\title{
Sludge Source Term (U) (PUREX Process Radionuclide Dose Impact)
}

by

C. I. Aponte

Westinghouse Savannah River Company

Savannah River Site

Aiken, South Carolina 29808

DOE Contract No. DE-AC09-89SR18035

This paper was prepared in connection with work done under the above contract number with the U.S.

Department of Energy. By acceptance of this paper, the publisher and/or recipient acknowledges the U.S. Government's right to retain a nonexclusive, royalty-free license in and to any copyright covering this paper, along with the right to reproduce and to authorize others to reproduce all or part of the copyrighted paper. 


\section{DISCLAIMER}

Portions of this document may be illegible in electronic image products. Images are produced from the best available original document. 


\section{DISCLAIMER}

This report was prepared as an account of work sponsored by an agency of the United States Government. Neither the United States Government nor any agency thereof, nor any of their employees, makes any warranty, express or implied, or assumes any legal liability or responsibility for the accuracy, completeness, or usefuiness of any information, apparatus, product, or process disclosed, or represents that its use would not infringe privately owned rights. Reference herein to any specific commercial product, process, or service by trade name, trademark, manufacturer, or otherwise does not necessarily constitute or imply its endorsement, recommendation, or favoring by the United States Government or any agency thereof. The views and opinions of authors expressed herein do not necessarily state or reflect those of the United States Government or any agency thereof.

This report has been reproduced directly from the best available copy.

Available to DOE and DOE contractors from the Office of Scientific and Technical Information, P.O. Box 62, Oak Ridge, TN 37831; prices available from (615) 576-8401.

Available to the public from the National Technical Information Service, U.S. Department of Commerce, 5285 Port Royal Road, Springfield, VA 22161. 


\section{HIGH LEVEL WASTE ENGINEERING}

HIGH LEVEL WASTE ENGINEERING SUPPORT

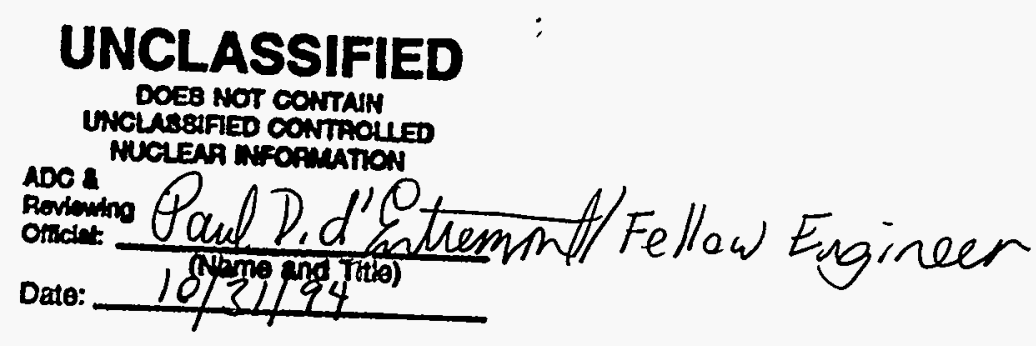

SLUDGE SOURCE TERM (U)

(PUREX Process Radionuclide Dose Impact)

BY

C. I. Aponte

June 28, 1994
WSRC-TR-94-0262

Revision : 0

KEYWORDS:

Fuel Assemblies

Mark 31A, 31B

Dose Calculations

Inhalation, Ingestion

Safety Analysis

\section{RETENTION:}

Permanent

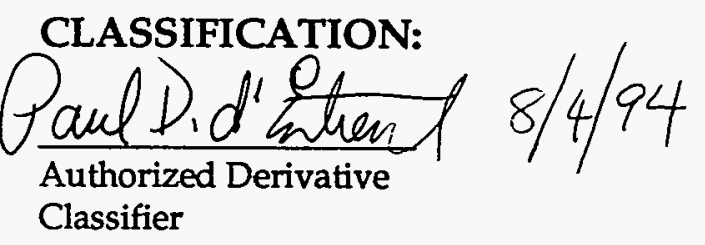

\section{Ql. Gearte}

C. I. Aponte, Author, HLWE Mich

M. C. Chandler, Manager, HLWE Technology Studies CE. Bess

C. E. Bess, Technical Reviewer

MClende

J. R. Chandler, Technical Reviewer

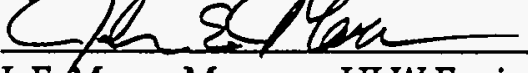

J. E.Marma, Manager, HLW Engineering Support

C) Hnickert tor

T. M. Monahon, Manager, HLW Engineering
Date: $6 / 20 / 94$

Date: $7 / 26 / 94$

Date: $6 / 29 / 94$

Date: $7 / 20 / 54$

Date: $\frac{8 / 8 / 94}{9 / 21 / 94}$ 


\section{TABLE OF CONTENT}

LIST of ILLUSTRATIONS....................................................................................

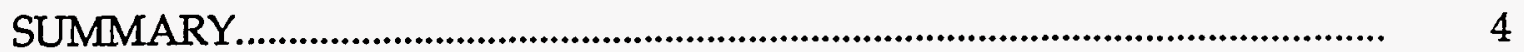

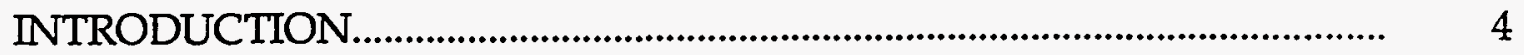

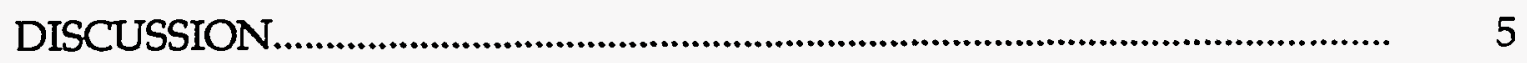

SAMPLE CALCULATION.........................................................................

ANALYSIS..................................................................................................... 9

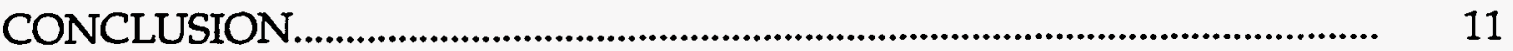

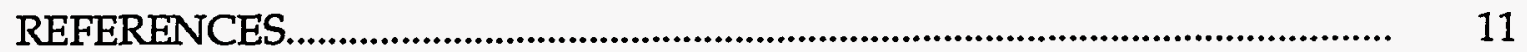

APPENDIX A

PUREX Radionuclide Dose Contribution (Inhalation)........................................... 12

APPENDIX B

PUREX Radionuclide Dose Contribution (Ingestion)............................................ 20

APPENDIX C

Radionuclide Dose Contribution Comparison............................................... 28 
WSRC-TR-94-0262

Revision 0

Page 3 of 31

\section{ILLUSTRATIONS}

\section{TABLES}

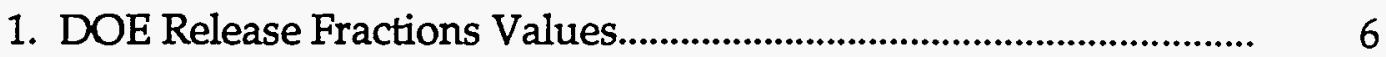

2. Inhalation Radionuclide Dose Contribution........................................ 9

3. Radionuclide Dose Contribution (Ingestion)....................................... 10 


\section{SUMMARY}

This report analyzes the radionuclide dose impact of the PUREX process waste stream. The radionuclide ingestion and inhalation pathways are analyzed. Two spent fuel assemblies processed in the Separation facilities are analyzed, the Mark 31A and Mark 31B.

The individual radionuclide significance to dose is evaluated in terms of dose percentage. Comparing the radionuclide individual dose value allows the determination of those radionuclides whose dose impact is significant.

The results of this analysis demonstrate that a limited number of radionuclides contribute $1 \%$ or more to the total dose and that the major contributor to the sludge source dose is strontium.

The results obtained permit reducing the list of radionuclides to be considered in the development of source terms to support the High Level Waste Safety Analysis Report.

\section{INTRODUCTION}

The PUREX process has been used in the Separation facilities in F \& H-Area. It has been used in the F-Canyon since building start up in November 1954 to separate plutonium from uranium. In the $\mathrm{H}$-Canyon building it was used between August 1955 and March 1959 to separate weapons grade plutonium from uranium.

This report analyzes both the inhalation and ingestion radionuclide dose impact of the PUREX Process waste stream. The spent fuel assemblies analyzed are the Mark 31A and Mark 31B.

The results from this report will be used to establish source terms for High Level Waste. This report summarizes the approach used to determine those radionuclides whose dose impact is significant for establishing the sludge source terms. 
WSRC-TR-94-0262

Revision 0

Page 5 of 31

\section{DISCUSSION}

\section{Isotopic Dose Calculations}

The data for the Mark 31A and Mark 31B and the ICRP-30 (Ref. 1) Dose Conversion Factors were used to determine the isotopic source dose contribution in terms of rem.

Only those radionuclides with a half life exceeding a day were considered. The isotopic dose contribution was evaluated at 5 and 15 years for each spent fuel assembly (Mark 31A and Mark 31B).

The data was normalized in terms of Pu-239. The spent fuel isotopic content was adjusted using the data from Ref. 3 . From this data the fraction insoluble was calculated as (1.0 - soluble fraction) and used to account for the amount of each radionuclide in the sludge. In order to determine those radionuclides whose dose impact is important, the radionuclide source values were converted to dose values. To obtain the dose contribution for each isotope in terms of rem, the normalized isotopic content for each isotope was multiplied by the ICRP-30 (Ref. 1) Inhalation and Ingestion Dose Conversion factors. For both the ingestion and the inhalation pathways, the calculated dose values were added together to determine the total dose (rem). The individual isotope inhalation and ingestion dose contribution was calculated in terms of dose percentage.

The isotopic dose contribution for the inhalation pathway was also evaluated considering evaporative conditions. Table 1 contains the Release Fractions values used to adjust the Dose Conversion factors (for evaporative conditions) as specified by DOE (Ref. 1). No Release Fractions values are applied to volatile conditions. 
WSRC-TR-94-0262

Revision 0

Page 6 of 31

\section{TABLE 1}

DOE Release Fractions Values

1. Gases -

(such as tritium, krypton, xenon, argon, radon, chlorine, etc.).

2. Highly volatile/combustible (phosphorus, sulfur, potassium, iodine, sodium, bromine)

3. Semi-volatile (selenium, mercury, cesium, polonium, tellurium ruthenium, carbon)

\section{Solid/Powder/Liquid}

(All materials not listed above.)

1.0

0.5

$1 \mathrm{E}-2$

$1 E-3$

The results of the calculations are tabulated in Appendix A and Appendix B for the inhalation and the ingestion pathway, respectively. A radionuclide contributing at least one percent of the total dose is considered significant to the source dose. Those isotopes contributing one percent or more to the total dose are indicated in bold-underlined face type. Tables 2 and 3 in the Analysis section summarize the dose contribution (dose percentage) for those radionuclides contributing one percent or more of the total dose for the inhalation and ingestion pathways. 
WSRC-TR-94-0262

Revision 0

Page 7 of 31

\section{SAMPLE CALCULATION}

For the Mark 31A spent fuel assembly at 5 years (radionuclide inhalation pathway):

\begin{tabular}{cc} 
Isotope & $\begin{array}{c}\text { Isotopic Content } \\
\text { (g/assembly) }\end{array}$ \\
\hline $\mathrm{H}-3$ & $3.64 \mathrm{E}-05$ \\
$:$ & $:$ \\
$\mathrm{Sr}-90$ & $2.16 \mathrm{E}+00$ \\
$:$ & $:$ \\
$\mathrm{Cs}-134$ & $6.39 \mathrm{E}-03$ \\
$\mathrm{Cs}-135$ & $5.91 \mathrm{E}-01$ \\
$\mathrm{Cs}-136$ & $6.85 \mathrm{E}-45$ \\
$\mathrm{Cs}-137$ & $4.59 \mathrm{E}+00$ \\
$:$ & $:$ \\
Pu-239 & $7.96 \mathrm{E}-01$ \\
$:$ & $:$
\end{tabular}

For each isotope the isotopic content was normalized in terms of Pu-239:

\begin{tabular}{cc} 
Isotope & $\begin{array}{c}\text { Normalized } \\
\text { Sludge Content }\end{array}$ \\
\hline $\mathrm{H}-3$ & $0.00 \mathrm{E}+00$ \\
$:$ & $:$ \\
$\mathrm{S}-90$ & $2.72 \mathrm{E}+00$ \\
$:$ & $:$ \\
$\mathrm{Cs}-134$ & $4.01 \mathrm{E}-04$ \\
$\mathrm{Cs}-135$ & $3.71 \mathrm{E}-02$ \\
$\mathrm{Cs}-136$ & $4.30 \mathrm{E}-46$ \\
$\mathrm{Cs}-137$ & $2.88 \mathrm{E}-01$ \\
$:$ & $:$ \\
Pu-239 & $1.00 \mathrm{E}+00$ \\
$:$ & $:$
\end{tabular}

Note:

- The isotopic content was adjusted by solubility fraction values to account for the amount of each radionuclide present in the sludge, Ref. 3. 
To express the source dose in terms of rem, the normalized isotopic content were multiplied by the ICRP-30 Inhalation Dose Conversion Factors. The dose values obtained are:

\begin{tabular}{ccc} 
Isotope & $\begin{array}{c}\text { Dose } \\
\text { (rem/g Pu-239) }\end{array}$ & $\begin{array}{c}\text { Percent } \\
\text { Dose }\end{array}$ \\
\hline $\mathrm{H}-3$ & $0.00 \mathrm{E}+00$ & $0 \%$ \\
$:$ & $\vdots$ & $:$ \\
$\mathrm{Sr}-90$ & $4.92 \mathrm{E}+08$ & $57 \%$ \\
$:$ & $\vdots$ & $:$ \\
$\mathrm{Cs}-134$ & $2.44 \mathrm{E}+04$ & $0 \%$ \\
$\mathrm{Cs}-135$ & $1.92 \mathrm{E}-01$ & $0 \%$ \\
$\mathrm{Cs}-136$ & $2.35 \mathrm{E}-37$ & $0 \%$ \\
$\mathrm{Cs}-137$ & $3.36 \mathrm{E}+05$ & $0 \%$ \\
$:$ & $:$ & $:$ \\
Pu-239 & $3.16 \mathrm{E}+07$ & $4 \%$ \\
$:$ & $:$ & $:$ \\
\cline { 2 - 2 } & Total 8.25E+08 &
\end{tabular}

The individual isotope dose value was then divided by the total dose to determine the isotopic dose contribution.

To determine the radionuclide contribution considering evaporative conditions (for the inhalation pathway only) the ICRP-30 Dose Conversion Factors were adjusted by the DOE release fraction values.

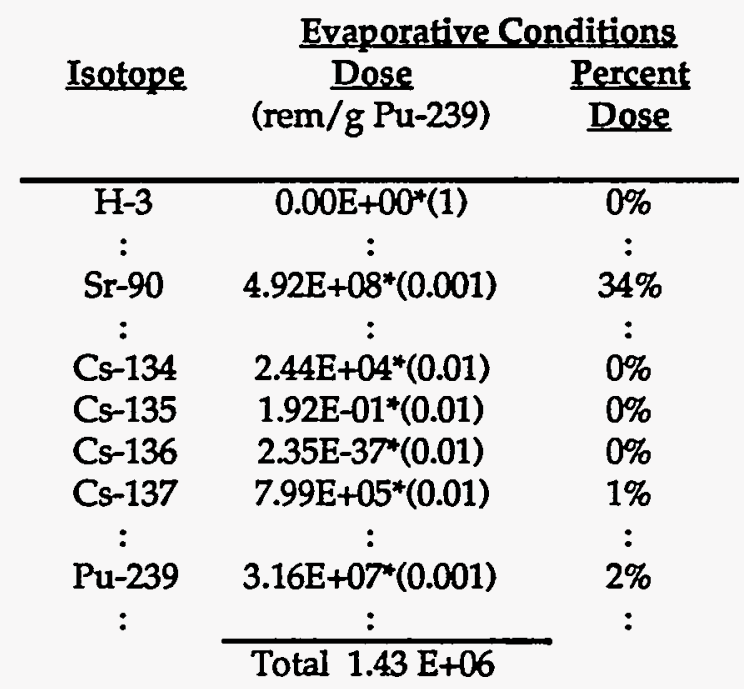

Note:

- The same above calculations were performed for the ingestion pathway. 


\section{ANALYSIS}

Tables 2 \& 3 summarize the dose percentage of those radionuclides contributing one percent or more to the total dose for the inhalation and ingestion pathways. The results included in Appendix A, Appendix B, and Tables 2 \& 3 demonstrate that only a limited number of radionuclides have a significant impact to the total dose.

TABLE 2

RADIONUCLIDE.INHALATTONDOSE CONTRIBUTION

(Dose Percentage)

\begin{tabular}{|c|c|c|c|c|c|c|c|c|}
\hline & \multicolumn{4}{|c|}{ Volatile Conditions } & \multicolumn{4}{|c|}{ Evaporative Conditions } \\
\hline & \multicolumn{2}{|c|}{5 years } & \multicolumn{2}{|c|}{15 years } & \multicolumn{2}{|c|}{5 years } & \multicolumn{2}{|c|}{15 years } \\
\hline & $31 \mathrm{~A}$ & 31B & $3 \overline{1 A}$ & $\overline{31 B}$ & 31A & $31 \mathrm{~B}$ & $31 \mathrm{~A}$ & $31 \mathrm{~B}$ \\
\hline Sr-90 & 60 & 62 & 8 & 68 & 祭 & 6 & 68 & $6 \%$ \\
\hline $\mathrm{Ru}-106$ & 4 & 4 & 0 & 0 & 28 & 28 & 0 & 0 \\
\hline Cs-137 & 0 & 0 & $\mathbf{0}$ & 0 & 1 & 1 & 1 & 1 \\
\hline Ce-144 & 8 & 8 & 0 & $\mathbf{0}$ & 6 & 6 & 0 & 0 \\
\hline Pm-147 & 2 & 2 & 0 & 0 & 2 & 2 & 0 & 0 \\
\hline Pu-239 & 4 & 4 & 5 & 5 & 3 & 3 & 5 & 5 \\
\hline $\mathrm{Pu}-240$ & 1 & 1 & 1 & 1 & 0 & 1 & 1 & 1 \\
\hline Pu-241 & 1 & 1 & 1 & 1 & 1 & 1 & 1 & 1 \\
\hline Am-241 & 20 & 17 & 28 & 24 & 15 & 13 & 27 & 24 \\
\hline
\end{tabular}


TABLE 3

RADIONUCLIDE DOSE

CONTRIBUTION

(Dose Percentage)

Sr-90

Y-90

$\mathrm{Ru}-106$

Cs-137

Ce-144

Pm-147

$\mathrm{Pu}-239$

Am-241

\begin{tabular}{|c|c|c|c|}
\hline \multicolumn{4}{|c|}{ INGESTION } \\
\hline \multicolumn{2}{|c|}{5 years } & \multicolumn{2}{|c|}{15 vears } \\
\hline $31 \mathrm{~A}$ & 31B & $3 \overline{1 A}$ & 31B \\
\hline 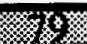 & $86 \%$ & 㫼 & 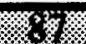 \\
\hline 6 & 6 & 7 & 7 \\
\hline 2 & 2 & 0 & 0 \\
\hline 2 & 2 & 2 & 2 \\
\hline 6 & 6 & 0 & 0 \\
\hline 1 & 1 & 0 & 0 \\
\hline 0 & 0 & 1 & 1 \\
\hline 2 & 2 & 3 & 3 \\
\hline
\end{tabular}

The major contributor to the total dose from the PUREX process sludge waste stream is Sr-90, under both the inhalation and ingestion pathway. Due to their short half-life as compared to the other radionuclides shown in Tables $2 \& 3, \mathrm{Ru}-$ $106, \mathrm{Ce}-144$ and $\mathrm{Pm}-147$ are significant to the dose only during a short time period. For the plutonium isotope, $\mathrm{Pu}-239$, it is shown that its contribution to the ingestion dose becomes significant after a long period of time.

Appendix $C$ presents the dose contribution for the inhalation and ingestion pathways in a comparative manner. From the Inh/Ing ratio column, it can be demonstrated that the cesium major dose impact occurs when evaluating the ingestion pathway. For the alpha emitters the major dose impact occurs through the inhalation pathway. 
WSRC-TR-94-0262

Revision 0

Page 11 of 31

\section{CONCLUSION}

Source terms can be established based on those radionuclides whose dose impact is significant. The individual radionuclide significance to dose can be readily determined if the source dose is evaluated in terms of rem.

The data analyzed demonstrate that a limited number of radionuclides is significant in determining source terms. Strontium-90 is the major contributor to the PUREX process sludge dose through both, the inhalation and the ingestion pathway.

\section{REFERENCES:}

1. DOE STANDARD, "Hazard Categorization and Accident Analysis Techniques for Compliance with DOE Order 5480.23, Nuclear Safety Analysis Reports", DOE-STD-1027-92, December 1992

2. DPSTD-80-39, "Preliminary Technical Data Summary - Defense Waste Processing Facility - Stage 2", December 1980

3. J. R. Fowler, "Update of Chemical and Radiochemical Composition of Decontaminated Soluble Waste from the Precipitation Process", DPST-82-759, August 6, 1982

4. M. C. Chandler, "Dose Contribution from High Level Waste Uranium and Plutonium (U)", WSRC-TR-93-604, November 9, 1993 


\section{PUREX RADIONUCLDE INHALATION DOSE CONTAIBUTION}

Mark 31A Fuot Assembly

5 yoars

Normalized Volatilecenditions Evaporative Conditions

Isotope Isotopic Content Sludge Content Dose Conversion Dose Percent Release Dose Percent (g/assembly) ( $g / g$ Pu-239) (ICRP-30, rem/g) (rem/g Pu-239) Dose Fraction Values (rem/g Pu-239) Dose

\begin{tabular}{|c|c|c|c|c|c|c|c|c|}
\hline $\mathrm{H}-3$ & 3.64E-05 & $0.00 \mathrm{E}+00$ & $9.22 E+05$ & $0.00 E+00$ & $0 \%$ & 1 & $0.00 E+00$ & $0 \%$ \\
\hline Cr-51 & $3.03 E-23$ & $3.61 E-23$ & $2.40 E+07$ & 8.66E-16 & $0 \%$ & 0.001 & 8.66E-19 & $0 \%$ \\
\hline Co- 60 & $4.50 E-03$ & $5.65 E-03$ & $1.70 E+08$ & $9.61 E+05$ & $0 \%$ & 0.001 & $9.61 E+02$ & $0 \%$ \\
\hline Se-79 & 2.51E-02 & 2.84E-02 & $6.20 E+02$ & $1.76 E+01$ & $0 \%$ & 0.01 & $1.76 \mathrm{E}-01$ & $0 \%$ \\
\hline $\mathrm{Rb}-87$ & $1.04 E+\infty 0$ & $6.54 E-02$ & 2.87E-04 & 1.88E-05 & $0 \%$ & 0.001 & 1.88E-08 & $0 \%$ \\
\hline Sr-89 & 3.87E-11 & $4.86 E-11$ & 1.07E+09 & 5.20E-02 & $0 \%$ & 0.001 & 5.20E-05 & $0 \%$ \\
\hline Sr-90 & $2.16 E+00$ & $2.72 E+\infty 0$ & $1.81 E+08$ & $4.92 E+08$ & $60 \%$ & 0.001 & $4.92 E+05$ & $44 \%$ \\
\hline$Y-90$ & 5.63E-04 & 7.07E-04 & $4.46 E+09$ & $3.15 E+06$ & $0 \%$ & 0.001 & $3.15 E+03$ & $0 \%$ \\
\hline Y-91 & 8.07E-10 & $1.01 E-09$ & $1.08 E_{+09}$ & $1.09 E+00$ & $0 \%$ & 0.001 & $1.09 E-03$ & $0 \%$ \\
\hline Zr-93 & $3.05 E+00$ & $3.83 E+00$ & $8.04 E+02$ & $3.08 E+03$ & $0 \%$ & 0.001 & $3.08 E+00$ & $0 \%$ \\
\hline $\mathrm{Zr}-95$ & $1.06 \mathrm{E}-08$ & 1.33E-08 & $3.86 E+08$ & $5.12 E+00$ & $0 \%$ & 0.001 & $5.12 E-03$ & $0 \%$ \\
\hline $\mathrm{Nb}-95$ & $1.22 \mathrm{E}-08$ & $1.53 E-08$ & $1.76 E+08$ & $2.70 E+00$ & $0 \%$ & 0.001 & 2.70E-03 & $0 \%$ \\
\hline $\mathrm{Nb}-95 \mathrm{~m}$ & 7.39E-12 & $9.28 E-12$ & $8.08 E+08$ & $7.50 E-03$ & $0 \%$ & 0.001 & $7.50 \mathrm{E}-06$ & $0 \%$ \\
\hline Tc-99 & $3.59 E+\infty 0$ & $0.00 E+00$ & 1.27E+02 & $0.00 E+00$ & $0 \%$ & 0.001 & $0.00 \mathrm{E}+00$ & $0 \%$ \\
\hline Ru-103 & $2.50 \mathrm{E}-14$ & 1.57E-14 & $2.51 E+08$ & 3.94E-06 & $0 \%$ & 0.01 & $3.94 E-08$ & $0 \%$ \\
\hline Bu-106 & $3.38 E-02$ & 2.12E-02 & $1.47 E+08$ & $3.12 E+07$ & $4 \%$ & 0.01 & $3.12 E+05$ & $28 \%$ \\
\hline Pd-107 & 7.27E-01 & 9.13E-01 & $6.68 E+00$ & $6.10 E+\infty 0$ & $0 \%$ & 0.001 & $6.10 E-03$ & $0 \%$ \\
\hline Cd-115m & 2.14E-15 & 2.55E-15 & $1.65 E+09$ & 4.21E-06 & $0 \%$ & 0.001 & 4.21E-09 & $0 \%$ \\
\hline $\ln -115$ & 4.24E-02 & 5.06E-02 & 1.77E-05 & 8.96E-07 & $0 \%$ & 0.001 & 8.96E-10 & $0 \%$ \\
\hline Sn-121m & 1.17E-05 & 1.41E-05 & $3.48 E+05$ & $4.91 E+00$ & $0 \%$ & 0.001 & 4.91E-03 & $0 \%$ \\
\hline Sn-123 & $1.25 E-06$ & 1.51E-06 & $2.47 E+08$ & $3.74 E+02$ & $0 \%$ & 0.001 & 3.74E-01 & $0 \%$ \\
\hline Sn-125 & $1.10 E-59$ & 1.32E-59 & $1.52 E+09$ & $2.01 E-50$ & $0 \%$ & 0.001 & $2.01 E-53$ & $0 \%$ \\
\hline Sn-126 & $1.20 \mathrm{E}-01$ & $1.45 E-01$ & $2.44 E+03$ & $3.53 E+02$ & $0 \%$ & 0.001 & 3.53E-01 & $0 \%$ \\
\hline$S b-124$ & $6.08 E-14$ & 7.59E-14 & $3.67 E+08$ & 2.79E-05 & $0 \%$ & 0.001 & 2.79E-08 & $0 \%$ \\
\hline Sb-125 & 2.39E-02 & $2.98 E-02$ & $1.01 E+07$ & $3.01 E+05$ & $0 \%$ & 0.001 & $3.01 E+02$ & $0 \%$ \\
\hline$S b-126$ & 5.70E-10 & $6.87 E-10$ & $8.36 E+08$ & 5.75E-01 & $0 \%$ & 0.001 & 5.75E-04 & $0 \%$ \\
\hline To-127m & 3.16E-07 & 3.96E-07 & $1.79 E+08$ & $7.09 E+01$ & $0 \%$ & 0.01 & 7.09E-01 & $0 \%$ \\
\hline To-129m & $4.41 E-18$ & 5.54E-18 & $6.03 E+08$ & 3.34E-09 & $0 \%$ & 0.01 & $3.34 E-11$ & $0 \%$ \\
\hline |-129 & 7.85E-01 & $0.00 E+\infty$ & $3.18 E+01$ & $0.00 E+00$ & $0 \%$ & 0.5 & $0.00 \mathrm{E}+\infty 0$ & $0 \%$ \\
\hline $\mid-131$ & 2.81E-69 & $0.00 E+\infty$ & $3.97 E+09$ & $0.00 E+\infty$ & $0 \%$ & 0.5 & $0.00 E+\infty$ & $0 \%$ \\
\hline Cs-134 & $6.39 E-03$ & 4.01E-04 & $6.08 E+07$ & $2.44 E+04$ & $0 \%$ & 0.01 & $2.44 E+02$ & $0 \%$ \\
\hline Cs-135 & $5.91 E-01$ & 3.71E-02 & $5.18 E+\infty$ & $1.92 E-01$ & $0 \%$ & 0.01 & $1.92 E-03$ & $0 \%$ \\
\hline Cs-136 & $6.85 E-45$ & $4.30 E-46$ & $5.47 E+08$ & 2.35E-37 & $0 \%$ & 0.01 & 2.35E-39 & $0 \%$ \\
\hline Cs-137 & $4.59 E+00$ & 2.88E-01 & 2.77E+06 & $7.99 E+05$ & $0 \%$ & 0.01 & $7.99 E+03$ & $1 \%$ \\
\hline $\mathrm{Ba}-140$ & $1.86 \mathrm{E}-43$ & $2.33 E-43$ & $2.63 E+08$ & $6.14 E-35$ & $0 \%$ & 0.001 & $6.14 E-38$ & $\overline{0 \%}$ \\
\hline La-140 & $2.80 E-44$ & $3.52 E-44$ & $2.45 E+00$ & 8.62E-35 & $0 \%$ & 0.001 & $8.62 E-38$ & $0 \%$ \\
\hline Co-141 & 3.72E-17 & 4.67E-17 & $2.42 E+08$ & 1.13E-08 & $0 \%$ & 0.001 & 1.13E-11 & $0 \%$ \\
\hline Co-144 & $4.60 \mathrm{E}-02$ & $5.78 E-02$ & $1.12 E+09$ & $6.47 E+07$ & $8 \%$ & 0.001 & $6.47 E+04$ & $6 \%$ \\
\hline$P r-143$ & $6.19 E-41$ & 7.77E-41 & $4.92 E+08$ & 3.82E-32 & $0 \%$ & 0.001 & 3.82E-35 & $0 \%$ \\
\hline Nd-144 & $4.25 E+00$ & $5.34 E+00$ & $0.00 E+\infty 0$ & $0.00 E+\infty$ & $0 \%$ & 0.001 & $0.00 E+\infty 0$ & $0 \%$ \\
\hline Nd-147 & 5.90E-51 & 7.41E-51 & $5.02 E+08$ & $3.72 E-42$ & $0 \%$ & 0.001 & 3.72E-45 & $0 \%$ \\
\hline Pm-147 & 4.82E-01 & 6.05E-01 & $3.15 E+07$ & $1.91 E+07$ & $2 \%$ & 0.001 & $1.91 E+04$ & $2 \%$ \\
\hline Pm-148 & 6.65E-18 & $8.35 E-18$ & $1.64 E+08$ & 1.37E-08 & $0 \%$ & 0.001 & 1.37E-11 & $\overline{0 \%}$ \\
\hline Pm-148m & 7.41E-16 & 9.31E-16 & $2.14 E+08$ & $1.99 E-07$ & $0 \%$ & 0.001 & $1.99 E-10$ & $0 \%$ \\
\hline Sm-147 & $1.33 E+\infty 0$ & $1.68 E+\infty 0$ & $1.65 \mathrm{E}+00$ & $2.76 E+\infty 0$ & $0 \%$ & 0.001 & 2.76E-03 & $0 \%$ \\
\hline Sm-151 & 2.59E-01 & 3.25E-01 & $7.63 E+05$ & $2.48 E+05$ & $0 \%$ & 0.001 & $2.48 E+02$ & $0 \%$ \\
\hline Eu-152 & 4.85E-04 & $6.09 E-04$ & $3.81 E+07$ & $2.32 E+04$ & $0 \%$ & 0.001 & $2.32 E+01$ & $0 \%$ \\
\hline Eu-154 & $1.03 E-02$ & 1.30E-02 & $6.86 E+07$ & $8.89 E+05$ & $0 \%$ & 0.001 & $8.89 E+02$ & $0 \%$ \\
\hline Eu-155 & 2.76E-02 & $3.46 E-02$ & $1.81 E+07$ & $6.26 E+0.5$ & $0 \%$ & 0.001 & $6.26 \mathrm{E}+02$ & $0 \%$ \\
\hline Eu-156 & 4.00E-38 & $5.02 E-38$ & $6.06 E+08$ & $3.04 E-29$ & $0 \%$ & 0.001 & $3.04 E-32$ & $0 \%$ \\
\hline Gd-152 & 1.75E-05 & 2.20E-05 & 5.15E-03 & 1.13E-07 & $0 \%$ & 0.001 & 1.13E-10 & $0 \%$ \\
\hline$T b-160$ & 2.12E-12 & $2.66 \mathrm{E}-12$ & $2.48 E+08$ & 6.61E-04 & $0 \%$ & 0.001 & 6.61E-07 & $0 \%$ \\
\hline Ra-224 & 4.31E-15 & 5.42E-15 & $4.67 E+11$ & 2.53E-03 & $0 \%$ & 0.001 & $2.53 E-06$ & $0 \%$ \\
\hline Th-228 & 8.36E-13 & $1.05 E-12$ & $2.54 E+11$ & 2.67E-01 & $0 \%$ & 0.001 & 2.67E-04 & $0 \%$ \\
\hline
\end{tabular}


PUREX RADIONUCLIDE INHALATION DOSE CONTRIBUTION

Mark 31A Fual Assombly

5 yoars

\begin{tabular}{|c|c|c|c|c|c|c|c|c|}
\hline \multirow[b]{2}{*}{ Isotopo } & \multirow{2}{*}{$\begin{array}{l}\text { Isotopic Content } \\
\text { (g/assembly) }\end{array}$} & \multirow{2}{*}{$\begin{array}{l}\text { Normalized } \\
\text { Sludge Content } \\
\text { (g/g Pu-239) }\end{array}$} & \multirow[b]{2}{*}{$\begin{array}{l}\text { Dose Comversion } \\
\text { (ICRP-30, rem/g) }\end{array}$} & \multicolumn{2}{|c|}{ Yolatile Conditions } & \multirow[b]{2}{*}{$\begin{array}{c}\text { Rolease } \\
\text { Fraction Values }\end{array}$} & \multicolumn{2}{|c|}{ Evaporative Conditions } \\
\hline & & & & $\begin{array}{l}\text { Dose } \\
\text { rem/g Pu-239) }\end{array}$ & $\begin{array}{c}\text { Percent } \\
\text { Dose }\end{array}$ & & $\begin{array}{c}\text { Dose } \\
\text { (rem/g Pu-239) }\end{array}$ & $\begin{array}{c}\text { Percent } \\
\text { Dose }\end{array}$ \\
\hline $\mathrm{U}-232$ & $1.56 \mathrm{E}-11$ & 1.95E-11 & $1.48 E+10$ & 2.89E-01 & $0 \%$ & 0.001 & $2.89 E-04$ & $0 \%$ \\
\hline$U-234$ & 1.42E-17 & $1.79 E-17$ & $8.08 E+05$ & $1.45 E-11$ & $0 \%$ & 0.001 & $1.45 E-14$ & $0 \%$ \\
\hline U-235 & $1.20 E-01$ & $1.51 E-01$ & $2.59 E+02$ & $3.90 E+01$ & $0 \%$ & 0.001 & 3.90E-02 & $0 \%$ \\
\hline U-236 & $3.74 E-03$ & 4. $\cong 9 E-03$ & $7.76 E+03$ & $3.64 E+01$ & $0 \%$ & 0.001 & $3.64 E-02$ & $0 \%$ \\
\hline U-238 & 7.03E+01 & $8.82 E+01$ & $4.03 E+01$ & $3.56 E+03$ & $0 \%$ & 0.001 & $3.56 E+00$ & $0 \%$ \\
\hline $\mathrm{Np}-237$ & $1.21 E-02$ & $1.52 E-02$ & $3.45 E+05$ & $5.25 E+03$ & $0 \%$ & 0.001 & $5.25 E+00$ & $0 \%$ \\
\hline Pu-236 & 8.01E-12 & $1.01 E-11$ & $8.53 E+10$ & 8.58E-01 & $0 \%$ & 0.001 & 8.58E-04 & $0 \%$ \\
\hline Pu-238 & $1.26 E-04$ & $1.58 E-04$ & $7.88 E+09$ & $1.25 E+06$ & $0 \%$ & 0.001 & $1.25 E+03$ & $0 \%$ \\
\hline$P_{u}=239$ & $7.96 \mathrm{E}-01$ & $1.00 \mathrm{E}+00$ & $3.16 E+07$ & $3.16 E+07$ & $4 \%$ & 0.001 & $3.16 E+04$ & $3 \%$ \\
\hline Pu-240 & 4.59E-02 & $5.76 E-02$ & $1.16 \mathrm{E}+08$ & $6.68 E+08$ & $\overline{1 x}$ & 0.001 & $6.68 E+03$ & $0 \%$ \\
\hline Ru-241 & 5.12E-03 & 6.43E-03 & $1.03 E+09$ & $6 . œ E+06$ & $1 \%$ & 0.001 & $6.62 E+03$ & $1 \%$ \\
\hline$\overline{P u-242}$ & 2.13E-04 & 2.68E-04 & $1.89 E+06$ & $5.06 E+02$ & $\overline{0 \%}$ & 0.001 & $5.06 E-01$ & $\overline{0 \%}$ \\
\hline$A m-241$ & 7.34E-02 & 9.21E-02 & $1.79 E+09$ & $1.65 E+08$ & $20 \%$ & 0.001 & $1.65 \mathrm{E}+05$ & $15 \%$ \\
\hline Am-242m & $4.00 \mathrm{E}-05$ & 5.03E-0.5 & $4.96 E+09$ & $2.49 E+0.5$ & $0 \%$ & 0.001 & $2.49 E+02$ & $0 \%$ \\
\hline Am-243 & $4.05 E-06$ & $5.08 E-06$ & $1.04 E+08$ & $5.29 E+02$ & $0 \%$ & 0.001 & 5.29E-01 & $0 \%$ \\
\hline $\mathrm{Cm}-242$ & $2.09 \mathrm{E}-07$ & 2.63E- 07 & $5.62 E+10$ & $1.48 E+04$ & $0 \%$ & 0.001 & $1.48 E+01$ & $0 \%$ \\
\hline $\mathrm{Cm}-243$ & $4.89 E-07$ & $6.14 E-07$ & $1.81 E+10$ & $1.11 E+04$ & $0 \%$ & 0.001 & 1.11E+01 & $0 \%$ \\
\hline Cm-244 & $6.53 E-08$ & 8.20E-08 & $2.18 E+10$ & $1.79 E+03$ & $0 \%$ & 0.001 & $1.79 E+00$ & $0 \%$ \\
\hline $\mathrm{Cm}-245$ & 3.72E-10 & 4.67E-10 & 9.27E+07 & 4.33E-02 & $0 \%$ & 0.001 & 4.33E-0.5 & $0 \%$ \\
\hline $\mathrm{Cm}-246$ & $3.51 E-12$ & 4.41E-12 & $1.65 E+08$ & 7.28E-04 & $0 \%$ & 0.001 & 7.28E-07 & $0 \%$ \\
\hline $\mathrm{Cm}-247$ & $3.66 \mathrm{E}-15$ & $4.60 \mathrm{E}-15$ & $4.55 E+04$ & $2.09 E-10$ & $0 \%$ & 0.001 & $2.09 E-13$ & $0 \%$ \\
\hline $\mathrm{Cm}-248$ & 1.93E-17 & 2.42E-17 & $8.08 E+06$ & $1.95 E-10$ & $0 \%$ & 0.001 & $1.95 E-13$ & $0 \%$ \\
\hline Bk-249 & $1.06 \mathrm{E}-23$ & 1.33E-23 & 2.17E+09 & $2.88 E-14$ & $0 \%$ & 0.001 & $2.88 E-17$ & $0 \%$ \\
\hline Cf-249 & $5.80 \mathrm{E}-22$ & $7.28 \mathrm{E}-22$ & $2.20 E+09$ & $\frac{1.60 E-12}{8.25 E+08}$ & $0 \%$ & 0.001 & $\frac{1.60 E-15}{1.11 E+06}$ & $0 \%$ \\
\hline
\end{tabular}




\section{PUREX RADIONUCLIDE INHALATION DOSE CONTRIBUTION}

Mark 31A Fuol Assombly

15 years

Normalized Volatile Conditions

Isotope Isotopic Content Sludge Content Dose Conversion Dose Percent (g/assembly) ( $(g / g$ Pu-239) (ICRP-30, rem/g) (rem/g Pu-239) Dose
Evaporative Conditions Release Dose Percent Fraction Values (rem/g Pu-239) Dose

\begin{tabular}{|c|c|c|c|c|c|c|c|c|}
\hline $\mathrm{H}-3$ & 2.09E-05 & $0.00 E+00$ & $9.22 E+05$ & $0.00 E+00$ & $0 \%$ & 1 & $0.00 E+00$ & $0 \%$ \\
\hline Cr-51 & 8.51E-63 & $1.02 E-62$ & $2.40 E+07$ & 2.44E-55 & $0 \%$ & 0.001 & 2.44E-58 & $0 \%$ \\
\hline Co- 60 & $1.20 \mathrm{E}-03$ & $1.51 E-03$ & $1.70 E+08$ & $2.57 E+05$ & $0 \%$ & 0.001 & $2.57 E+02$ & $0 \%$ \\
\hline Se-79 & 2.51E-02 & 2.84E-02 & $6.20 E+02$ & $1.76 \mathrm{E}+01$ & $0 \%$ & 0.01 & 1.76E-01 & $0 \%$ \\
\hline$R b-87$ & $1.04 E+00$ & 6.54E-02 & 2.87E-04 & 1.88E-05 & $0 \%$ & 0.001 & $1.88 E-08$ & $0 \%$ \\
\hline Sr-89 & 2.78E-32 & $3.49 E-32$ & $1.07 E+09$ & 3.73E-23 & $0 \%$ & 0.001 & 3.73E-26 & $0 \%$ \\
\hline Sr-90 & $1.69 E+00$ & $2.12 E+00$ & $1.81 E+08$ & $3.85 \mathrm{E}+08$ & $64 \%$ & 0.001 & $3.85 E+05$ & $63 \%$ \\
\hline$Y-90$ & 4.40E-04 & 5.53E-04 & $4.46 E+09$ & $2.46 E+06$ & $0 \%$ & 0.001 & $2.46 \mathrm{E}+03$ & $0 \%$ \\
\hline$Y-91$ & 1.39E-28 & 1.75E-28 & $1.08 E+09$ & $1.89 E-19$ & $0 \%$ & 0.001 & 1.89E-22 & $0 \%$ \\
\hline Zr-93 & $3.04 E+00$ & $3.82 E+00$ & $8.04 E+02$ & $3.07 E+03$ & $0 \%$ & 0.001 & $3.07 E+00$ & $0 \%$ \\
\hline $\mathrm{Zr}-95$ & 1.73E-25 & 2.17E-25 & $3.86 \mathrm{E}+0.8$ & 8.37E-17 & $0 \%$ & 0.001 & 8.37E-20 & $0 \%$ \\
\hline Nb-95 & 1.99E-25 & 2.51E-25 & $1.76 \mathrm{E}+08$ & 4.41E-17 & $0 \%$ & 0.001 & 4.41E-20 & $0 \%$ \\
\hline $\mathrm{Nb}-95 \mathrm{~m}$ & 1.21E-28 & 1.52E-28 & $8.08 E+08$ & 1.23E-19 & $0 \%$ & 0.001 & 1.23E-22 & $0 \%$ \\
\hline Tc-99 & $3.59 E+00$ & $0.00 E+00$ & $1.27 E+02$ & $0.00 E+00$ & $0 \%$ & 0.001 & $0.00 E+\infty 0$ & $0 \%$ \\
\hline$R u-103$ & $4.29 E-42$ & $2.69 E-42$ & $2.51 E+08$ & $6.76 E-34$ & $0 \%$ & 0.01 & $6.76 E-36$ & $0 \%$ \\
\hline Ru-106 & $3.54 E-05$ & 2.23E-05 & $1.47 E+09$ & $3.27 E+04$ & $0 \%$ & 0.01 & $3.27 E+02$ & $0 \%$ \\
\hline Pd-107 & 7.27E-01 & 9.13E-01 & $6.68 E+\infty$ & $6.10 E+\infty 0$ & $0 \%$ & 0.001 & $6.10 E-03$ & $0 \%$ \\
\hline $\mathrm{Cd}-115 \mathrm{~m}$ & 4.76E-40 & $5.68 E-40$ & $1.65 E+09$ & 9.37E-31 & $0 \%$ & 0.001 & 9.37E-34 & $0 \%$ \\
\hline $\ln -115$ & 4.24E-02 & 5.07E-02 & 1.77E-05 & 8.97E-07 & $0 \%$ & 0.001 & 8.97E-10 & $0 \%$ \\
\hline $\mathrm{Sn}-121 \mathrm{~m}$ & $1.02 E-05$ & 1.23E-05 & $3.48 E+05$ & $4.28 E+\infty 0$ & $0 \%$ & 0.001 & 4.28E-03 & $0 \%$ \\
\hline Sn-123 & 3.76E-15 & $4.54 E-15$ & $2.47 E+08$ & $1.12 E-06$ & $0 \%$ & 0.001 & 1.12E-09 & $0 \%$ \\
\hline Sn-126 & 1.20E-01 & 1.45E-01 & $2.44 E+03$ & $3.53 E+02$ & $0 \%$ & 0.001 & 3.53E-01 & $0 \%$ \\
\hline Sb-124 & 3.31E-32 & 4.13E-32 & $3.67 E+08$ & 1.52E-23 & $0 \%$ & 0.001 & 1.52E-26 & $0 \%$ \\
\hline Sb-125 & $1.89 E-03$ & 2.36E-03 & $1.01 E+07$ & $2.38 E+0.4$ & $0 \%$ & 0.001 & $2.38 E+01$ & $0 \%$ \\
\hline Sb-126 & 5.70E-10 & $6.87 E-10$ & $8.36 \mathrm{E}+08$ & 5.75E-01 & $0 \%$ & 0.001 & $5.75 E-04$ & $0 \%$ \\
\hline Te-127m & $2.58 E-17$ & 3.24E-17 & $1.79 E+08$ & 5.80E-09 & $0 \%$ & 0.01 & 5.80E-11 & $0 \%$ \\
\hline To-129m & 5.31E-51 & 6.67E-51 & $6.03 E+08$ & $4.02 E-42$ & $0 \%$ & 0.01 & $4.02 E-44$ & $0 \%$ \\
\hline $\mid-129$ & 7.85E-01 & $0.00 E+00$ & $3.18 E+01$ & $0.00 E+00$ & $0 \%$ & 0.5 & $0.00 E+00$ & $0 \%$ \\
\hline Cs-134 & 2.90E-04 & 1.82E-05 & $6.08 E+07$ & $1.11 E+03$ & $0 \%$ & 0.01 & $1.11 E+01$ & $0 \%$ \\
\hline Cs-135 & 5.91E-01 & 3.71E-02 & $5.18 E+\infty$ & $1.92 E-01$ & $0 \%$ & 0.01 & $1.92 E-03$ & $0 \%$ \\
\hline C9-137 & $3.65 E+00$ & 2.29E-01 & 2.77E+06 & $6.35 E+05$ & $0 \%$ & 0.01 & $6.35 E+03$ & $1 \%$ \\
\hline$C_{\theta-141}$ & $5.90 E-51$ & $7.41 E-51$ & $2.42 E+08$ & $1.79 E-42$ & $0 \%$ & 0.001 & $1.79 E-45$ & $0 \%$ \\
\hline Co-144 & $6.26 E-06$ & $7.87 E-06$ & $1.12 E+09$ & $8.81 E+03$ & $0 \%$ & 0.001 & $8.81 E+\infty 0$ & $0 \%$ \\
\hline Nd-144 & $4.30 E+00$ & $5.40 E+\infty 0$ & $0.00 \mathrm{E}+00$ & $0.00 E+\infty 0$ & $0 \%$ & 0.001 & $0.00 E+\infty$ & $0 \%$ \\
\hline Pm-147 & 3.43E-02 & 4.31E-02 & $3.15 E+07$ & $1.36 \mathrm{E}+06$ & $0 \%$ & 0.001 & $1.36 \mathrm{E}+03$ & $0 \%$ \\
\hline Pm-148 & $1.59 E-44$ & $1.99 E-44$ & $1.64 E+09$ & 3.27E-35 & $0 \%$ & 0.001 & 3.27E-38 & $0 \%$ \\
\hline $\mathrm{Pm}-148 \mathrm{~m}$ & $1.77 E-42$ & $2.22 E-42$ & $2.14 E+08$ & $4.75 E-34$ & $0 \%$ & 0.001 & 4.75E-37 & $0 \%$ \\
\hline $\mathrm{Sm}-147$ & $1.78 E+00$ & $2.24 E+\infty$ & $1.65 E+\infty 0$ & $3.69 E+\infty 0$ & $0 \%$ & 0.001 & 3.69E-03 & $0 \%$ \\
\hline Sm-151 & $2.40 \mathrm{E}-01$ & $3.02 E-01$ & $7.63 E+05$ & $2.30 E+0.5$ & $0 \%$ & 0.001 & $2.30 E+02$ & $0 \%$ \\
\hline Eu-152 & 2.84E-04 & 3.57E-04 & $3.81 E+07$ & $1.36 E+04$ & $0 \%$ & 0.001 & $1.36 E+01$ & $0 \%$ \\
\hline Eu-154 & $4.61 E-03$ & 5.79E-03 & $6.86 E+07$ & $3.97 E+05$ & $0 \%$ & 0.001 & $3.97 E+02$ & $0 \%$ \\
\hline Eu-155 & 6.50E-03 & $8.16 E-03$ & $1.81 E+07$ & $1.48 E+05$ & $0 \%$ & 0.001 & $1.48 E+02$ & $0 \%$ \\
\hline Gd-152 & $1.75 E-05$ & 2.20E-0.5 & 5.15E-03 & 1.13E-07 & $0 \%$ & 0.001 & 1.13E-10 & $0 \%$ \\
\hline $\mathrm{Tb}-160$ & 1.32E-27 & 1.65E-27 & $2.48 E+08$ & 4.10E-19 & $0 \%$ & 0.001 & 4.10E-22 & $0 \%$ \\
\hline Ra-224 & 2.81E-15 & $3.53 E-15$ & 4.67E+11 & $1.65 E-03$ & $0 \%$ & 0.001 & $1.65 E-06$ & $0 \%$ \\
\hline Th-228 & $5.46 \mathrm{E}-13$ & 6.86E-13 & $2.54 E+11$ & $1.74 E-01$ & $0 \%$ & 0.001 & $1.74 E-04$ & $0 \%$ \\
\hline U-232 & $2.08 E-11$ & 2.ळE-11 & $1.48 E+10$ & 3.87E-01 & $0 \%$ & 0.001 & 3.87E-04 & $0 \%$ \\
\hline U-234 & 1.42E-17 & 1.79E-17 & $8.08 E+05$ & $1.45 E-11$ & $0 \%$ & 0.001 & $1.45 E-14$ & $0 \%$ \\
\hline$U-235$ & $1.20 E-01$ & 1.51E-01 & $2.59 E+02$ & $3.90 E+01$ & $0 \%$ & 0.001 & $3.90 \mathrm{E}-02$ & $0 \%$ \\
\hline U-236 & $3.74 E-03$ & $4.69 E-03$ & $7.76 E+03$ & $3.64 E+01$ & $0 \%$ & 0.001 & 3.64E-02 & $0 \%$ \\
\hline U-238 & 7.03E+01 & $8.82 E+01$ & $4.03 E+01$ & $3.56 E+03$ & $0 \%$ & 0.001 & $3.56 \mathrm{E}+00$ & $0 \%$ \\
\hline Np-237 & $1.21 E-02$ & 1.52E-02 & $3.45 E+05$ & $5.25 E+03$ & $0 \%$ & 0.001 & $5.25 E+00$ & $0 \%$ \\
\hline Pu-236 & $7.05 E-13$ & 8.85E-13 & $8.53 E+10$ & $7.55 E-02$ & $0 \%$ & 0.001 & 7.55E-05 & $0 \%$ \\
\hline Pu-238 & $1.18 E-04$ & $1.48 E-04$ & $7.88 E+09$ & 1.17E+06 & $0 \%$ & 0.001 & $1.17 E+03$ & $0 \%$ \\
\hline $\mathrm{Pu}-239$ & 7.96E-01 & $1.00 E+\infty 0$ & $3.16 E+07$ & $3.16 E+07$ & $5 \%$ & 0.001 & $3.16 E+04$ & $5 \%$ \\
\hline
\end{tabular}




\section{PUREX RADIONUCLIDE INHALATION DOSE CONTRIBUTION}

Mark 31A Fuol Assembly

15 yoars

Normalized

Isotope Isotopic Content Sludge Content (g/assembly) (g/g Pu-239)
Volatile Conditions

Dose Percent

(ICRP-30, rem/g) (rem/g Pu-239) Dose
Release
raction Valu Fraction Values

\begin{tabular}{lllllllll}
\hline Pu-240 & $4.58 E-02$ & $5.75 E-02$ & $1.16 E+08$ & $6.67 E+06$ & $1 \%$ & 0.001 & $6.67 E+03$ & $1 \%$ \\
Pu-241 & $3.20 E-03$ & $4.01 E-03$ & $1.03 E+09$ & $4.13 E+06$ & $1 \%$ & 0.001 & $4.13 E+03$ & $1 \%$ \\
Pu-242 & $2.14 E-04$ & $2.68 E-04$ & $1.89 E+06$ & $5.07 E+02$ & $0 \%$ & 0.001 & $5.07 E-01$ & $0 \%$ \\
Am-241 & $7.41 E-02$ & $9.31 E-02$ & $1.79 E+09$ & $1.67 E+08$ & $28 \%$ & 0.001 & $1.67 E+05$ & $27 \%$ \\
Am-242m & $3.82 E-05$ & $4.80 E-05$ & $4.96 E+09$ & $2.38 E+05$ & $0 \%$ & 0.001 & $2.38 E+02$ & $0 \%$ \\
Am-243 & $4.04 E-06$ & $5.08 E-06$ & $1.04 E+08$ & $5.28 E+02$ & $0 \%$ & 0.001 & $5.28 E-01$ & $0 \%$ \\
Cm-242 & $9.26 E-08$ & $1.16 E-07$ & $5.62 E+10$ & $6.54 E+03$ & $0 \%$ & 0.001 & $6.54 E+00$ & $0 \%$ \\
Cm-243 & $3.84 E-07$ & $4.82 E-07$ & $1.81 E+10$ & $8.72 E+03$ & $0 \%$ & 0.001 & $8.72 E+00$ & $0 \%$ \\
Cm-244 & $4.45 E-08$ & $5.59 E-08$ & $2.18 E+10$ & $1.22 E+03$ & $0 \%$ & 0.001 & $1.22 E+00$ & $0 \%$ \\
Cm-245 & $3.72 E-10$ & $4.67 E-10$ & $9.27 E+07$ & $4.33 E-02$ & $0 \%$ & 0.001 & $4.33 E-05$ & $0 \%$ \\
Cm-246 & $3.51 E-12$ & $4.41 E-12$ & $1.65 E+08$ & $7.27 E-04$ & $0 \%$ & 0.001 & $7.27 E-07$ & $0 \%$ \\
Cm-247 & $3.66 E-15$ & $4.60 E-15$ & $4.55 E+04$ & $2.09 E-10$ & $0 \%$ & 0.001 & $2.09 E-13$ & $0 \%$ \\
Cm-248 & $1.93 E-17$ & $2.42 E-17$ & $8.08 E+06$ & $1.85 E-10$ & $0 \%$ & 0.001 & $1.95 E-13$ & $0 \%$ \\
Bk-249 & $3.33 E-27$ & $4.18 E-27$ & $2.17 E+09$ & $9.07 E-18$ & $0 \%$ & 0.001 & $9.07 E-21$ & $0 \%$ \\
Cf-249 & $5.79 E-22$ & $7.27 E-22$ & $2.20 E+09$ & $1.60 E-12$ & $0 \%$ & 0.001 & $1.60 E-15$ & $0 \%$ \\
& & & & $6.01 E+08$ & & & $6.07 E+05$
\end{tabular}

Evaporative Conditions Dose Percent Dose 


\section{PUREX RADIONUCLIDE INHALATION DOSE CONTRIBUTION}

Mark 31B Fual Assembly

5 yoars

Normalizod Volatile Cenditions

Isotope Isotopic Content Sludge Content Dose Conversion Dose Percent Release Evaperive Conditions $\begin{array}{llll}\text { (g/assembly) (g/g Pu-239) (ICRP-30, rem/g) (rem/g Pu-239) Dose Fraction Values (rem/g Pu-239) Dose } & \end{array}$

\begin{tabular}{|c|c|c|c|c|c|c|c|c|}
\hline$\overline{\mathrm{H}-3}$ & $3.10 E-05$ & $0.00 E+00$ & $9.22 E+05$ & $0.00 E+00$ & $0 \%$ & 1 & $0.00 E+00$ & $0 \%$ \\
\hline $\mathrm{Cr}-51$ & 1.03E-23 & 1.74E-23 & $2.40 E+07$ & 4.19E-16 & $0 \%$ & 0.001 & 4.19E-19 & $0 \%$ \\
\hline Co-60 & $1.53 E-03$ & 2.73E-03 & $1.70 E+08$ & $4.64 E+05$ & $0 \%$ & 0.001 & 4.64E+02 & $0 \%$ \\
\hline So-79 & 1.84E-02 & 2.96E-02 & $6.20 \mathrm{E}+02$ & $1.83 E+01$ & $0 \%$ & 0.01 & 1.83E-01 & $0 \%$ \\
\hline$R b-87$ & 7.83E-01 & $7.01 E-02$ & 2.87E-04 & 2.01E-05 & $0 \%$ & 0.001 & 2.01E-08 & $0 \%$ \\
\hline Si-89 & 3.11E-11 & 5.57E-11 & $1.07 E_{+} 09$ & 5.96E-02 & $0 \%$ & 0.001 & 5.96E-05 & $0 \%$ \\
\hline Sr-go & $1.63 E+\infty 0$ & $2.92 E+00$ & $1.81 E+08$ & $5.29 E+08$ & $62 \%$ & 0.001 & $5.29 E+05$ & $46 \%$ \\
\hline$Y-90$ & $4.25 E-04$ & $7.60 \mathrm{E}-0.4$ & $4.46 E+09$ & $3.39 E+06$ & $0 \%$ & 0.001 & $3.39 E+03$ & $0 \%$ \\
\hline$Y-91$ & $6.43 E-10$ & $1.15 E-09$ & $1.08 E+09$ & $1.24 E+00$ & $0 \%$ & 0.001 & $1.24 E-03$ & $0 \%$ \\
\hline Zr-93 & $2.28 E+00$ & $4.07 E+00$ & $8.04 E+02$ & $3.28 E+03$ & $0 \%$ & 0.001 & $3.28 E+00$ & $0 \%$ \\
\hline $\mathrm{Zr}-95$ & 8.27E-09 & $1.48 E-08$ & $3.86 E+08$ & $5.71 E+\infty 0$ & $0 \%$ & 0.001 & 5.71E-03 & $0 \%$ \\
\hline $\mathrm{Nb}-95$ & 9.56E-09 & 1.71E-08 & $1.76 E+08$ & $3.01 E+\infty 0$ & $0 \%$ & 0.001 & $3.01 E-03$ & $0 \%$ \\
\hline $\mathrm{Nb}-95 \mathrm{~m}$ & 5.79E-12 & $1.04 E-11$ & $8.08 E+08$ & 8.37E-03 & $0 \%$ & 0.001 & 8.37E-06 & $0 \%$ \\
\hline Tc-99 & $2.65 E+00$ & $0.00 E+00$ & $1.27 E+02$ & $0.00 E_{+}+0$ & $\infty$ & 0.001 & $0.00 E+00$ & $0 \%$ \\
\hline Ru-103 & $1.95 \mathrm{E}-14$ & $1.75 E-14$ & $2.51 E+08$ & 4.38E-06 & $0 \%$ & 0.01 & $4.38 E-08$ & $0 \%$ \\
\hline Bu-106 & $2.39 E-02$ & $2.14 E-02$ & $1.47 E+09$ & $3.14 E+07$ & $4 \%$ & 0.01 & $3.14 E+05$ & $28 \%$ \\
\hline Pd-107 & 5.12E-01 & 9.16E-01 & $6.68 E+00$ & $6.12 E+00$ & $0 \%$ & 0.001 & $6.12 E-03$ & $0 \%$ \\
\hline Cd-115m & $1.40 \mathrm{E}-15$ & 2.37E-15 & $1.65 \mathrm{E}+09$ & $3.92 E-06$ & $0 \%$ & 0.001 & 3.92E-09 & $0 \%$ \\
\hline $\ln -115$ & 2.60E-02 & 4.41E-02 & 1.77E-05 & 7.81E-07 & $0 \%$ & 0.001 & 7.81E-10 & $0 \%$ \\
\hline Sn-121m & 8.45E-06 & $1.45 E-05$ & $3.48 E+05$ & $5.05 E+\infty$ & $0 \%$ & 0.001 & $5.05 E-03$ & $0 \%$ \\
\hline Sn-123 & 8.05E-07 & 1.38E-06 & $2.47 E+08$ & $3.42 E+02$ & $0 \%$ & 0.001 & 3.42E-01 & $0 \%$ \\
\hline Sn-125 & 8.85E-60 & 1.52E-59 & $1.52 E+09$ & $2.31 E-50$ & $0 \%$ & 0.001 & 2.31E-53 & $0 \%$ \\
\hline Sn-126 & 7.99E-02 & 1.37E-01 & $2.44 E+03$ & $3.35 E_{+}+2$ & $0 \%$ & 0.001 & 3.35E-01 & $0 \%$ \\
\hline$S b-124$ & 3.56E-14 & $6.33 E-14$ & $3.67 E+08$ & 2.32E-05 & $0 \%$ & 0.001 & 2.32E-08 & $0 \%$ \\
\hline$S b-125$ & $1.55 E-02$ & 2.76E-02 & $1.01 E+07$ & $2.78 E+05$ & $0 \%$ & 0.001 & $2.78 E+02$ & $0 \%$ \\
\hline$S b-126$ & $3.80 E-10$ & $6.53 E-10$ & $8.36 E+08$ & $5.46 E-01$ & $0 \%$ & 0.001 & 5.46E-04 & $0 \%$ \\
\hline To-127m & 2.24E-07 & 4.01E-07 & $1.79 E+08$ & $7.18 E+01$ & $0 \%$ & 0.01 & 7.18E-01 & $0 \%$ \\
\hline To-129m & $3.45 E-18$ & $6.18 E-18$ & $6.03 E+08$ & 3.73E-09 & $0 \%$ & 0.01 & 3.73E-11 & $0 \%$ \\
\hline $1-129$ & 5.60E-01 & $0.00 E+\infty 0$ & $3.18 E+01$ & $0.00 E+\infty$ & $0 \%$ & 0.5 & $0.00 \mathrm{E}+00$ & $0 \%$ \\
\hline$|-13|$ & $2.56 E-69$ & $0.00 E+\infty 0$ & $3.97 E+09$ & $0.00 E+\infty$ & $0 \%$ & 0.5 & $0.00 \mathrm{E}+00$ & $0 \%$ \\
\hline Cs-134 & $5.01 E-03$ & 4.48E-04 & $6.08 E+07$ & $2.73 E+0.4$ & $0 \%$ & 0.01 & $2.73 E+02$ & $0 \%$ \\
\hline Cs-135 & 3.19E-01 & 2.86E-02 & $5.18 E+\infty$ & $1.48 E-01$ & $0 \%$ & 0.01 & $1.48 E-03$ & $0 \%$ \\
\hline Cs-136 & $5.78 E-45$ & 5.17E-46 & $5.47 E+08$ & $2.83 E-37$ & $0 \%$ & 0.01 & 2.83E-39 & $0 \%$ \\
\hline Cs-137 & $3.40 E+\infty$ & 3.04E-01 & $2.77 E+06$ & $8.42 E+05$ & $0 \%$ & 0.01 & $8.42 E+03$ & $1 \%$ \\
\hline $\mathrm{Ba}-140$ & $1.65 E-43$ & $2.96 E-43$ & $2.63 E+08$ & 7.78E-35 & $0 \%$ & 0.001 & 7.78E-38 & $\overline{0 \%}$ \\
\hline$L a-140$ & $2.49 E-44$ & 4.46E-44 & $2.45 E+09$ & $1.09 E-34$ & $0 \%$ & 0.001 & $1.09 E-37$ & $0 \%$ \\
\hline$C_{0-141}$ & $3.04 E-17$ & 5.44E-17 & $2.42 E+08$ & $1.32 E-08$ & $0 \%$ & 0.001 & $1.32 E-11$ & $0 \%$ \\
\hline Co-144 & 3.47E-02 & 6.21E-02 & $1.12 E+09$ & $6.96 E+07$ & $8 \%$ & 0.001 & $6.96 E+04$ & $6 \%$ \\
\hline Pr-143 & $5.50 E-41$ & $9.83 E-41$ & $4.92 E_{+08}$ & 4.83E-32 & $0 \%$ & 0.001 & $4.83 E-35$ & $0 \%$ \\
\hline Nd-144 & $3.17 E+\infty 0$ & $5.67 E+00$ & $0.00 E+\infty 0$ & $0.00 E+00$ & $0 \%$ & 0.001 & $0.00 E+00$ & $0 \%$ \\
\hline Nd-147 & $5.28 E-51$ & $9.44 E-51$ & $5.02 E+08$ & $4.74 E-42$ & $0 \%$ & 0.001 & $4.74 E-45$ & $0 \%$ \\
\hline Pm-147 & 3.63E-01 & 6.48E-01 & $3.15 E+07$ & $2.04 E+07$ & $2 \%$ & 0.001 & $2.04 E+04$ & $2 \%$ \\
\hline$P m-148$ & $3.66 \mathrm{E}-18$ & 6.54E-18 & $1.64 E+09$ & $1.07 E-08$ & $0 \%$ & 0.001 & 1.07E-11 & $\overline{0 \%}$ \\
\hline Pm-148m & 4.08E-16 & 7.29E-16 & $2.14 E+08$ & $1.56 E-07$ & $0 \%$ & 0.001 & $1.56 \mathrm{E}-10$ & $0 \%$ \\
\hline Sm-147 & $1.00 E+00$ & $1.79 E+00$ & $1.65 E+\infty 0$ & $2.95 E+00$ & $0 \%$ & 0.001 & $2.95 E-03$ & $0 \%$ \\
\hline $\mathrm{Sm}-151$ & $1.76 E-01$ & 3.15E-01 & $7.63 E+05$ & $2.40 E+05$ & $0 \%$ & 0.001 & $2.40 E+02$ & $0 \%$ \\
\hline Eu-152 & $3.77 E-04$ & 6.75E-04 & $3.81 E+07$ & $2.57 E+0.4$ & $0 \%$ & 0.001 & 2.57E+01 & $0 \%$ \\
\hline Eu-154 & $6.84 E-03$ & $1.22 E-02$ & $6.86 E+07$ & $8.39 E+05$ & $0 \%$ & 0.001 & $8.39 E+02$ & $0 \%$ \\
\hline Eu-155 & 1.86E-02 & 3.34E-02 & $1.81 E+07$ & $6.04 E+05$ & $0 \%$ & 0.001 & $6.04 E+02$ & $0 \%$ \\
\hline Eu-156 & 3.32E-38 & $5.94 E-38$ & $6.06 E+08$ & $3.60 E-29$ & $0 \%$ & 0.001 & $3.60 E-32$ & $0 \%$ \\
\hline Gd-152 & $1.06 E-0.5$ & 1.89E-05 & 5.15E-03 & $9.73 E-08$ & $0 \%$ & 0.001 & $9.73 E-11$ & $0 \%$ \\
\hline $\mathrm{Tb}-160$ & 1.21E-12 & 2.17E-12 & $2.48 E+08$ & 5.38E-04 & $0 \%$ & 0.001 & 5.38E-07 & $0 \%$ \\
\hline $\mathrm{Ra}-224$ & $5.05 E-11$ & $9.04 E-11$ & $4.67 E+11$ & $4.22 E+01$ & $0 \%$ & 0.001 & 4.22E-02 & $0 \%$ \\
\hline Th-228 & $9.79 E-09$ & $1.75 E-08$ & $2.54 E+11$ & $4.45 E+03$ & $0 \%$ & 0.001 & $4.45 E+\infty$ & $0 \%$ \\
\hline $\mathrm{U}-232$ & $1.82 E-07$ & 3.26E-07 & $1.48 E+10$ & $4.82 E+03$ & $0 \%$ & 0.001 & $4.82 E+00$ & $0 \%$ \\
\hline
\end{tabular}


PUREX RADIONUCLIDE INHALATION DOSE CONTRIBUTION

Mark 31B Fual Assambly 5 yoars

Normalized Volatile Conditions

Isotope Isotopic Content Sludge Content Dose Conversion Dose Percent (g/assembly) (g/g Pu-239) (ICRP-30, rem/g) (rem/g Pu-239) Dose

Release

Evaperative Conditions Fraction Values (rem/g Pu-239) Dose

\begin{tabular}{|c|c|c|c|c|c|c|c|c|}
\hline U-234 & $9.71 E-18$ & $1.74 E-17$ & B.08E+05 & $1.40 \mathrm{E}-11$ & $0 \%$ & 0.001 & $1.40 E-14$ & $0 \%$ \\
\hline U-235 & 7.95E-02 & 1.42E-01 & $2.59 E+02$ & $3.68 E+01$ & $0 \%$ & 0.001 & 3.68E-02 & $0 \%$ \\
\hline $\mathrm{U}-236$ & 2.75E-03 & 4.93E-03 & $7.76 E+03$ & $3.82 E+01$ & $0 \%$ & 0.001 & 3.82E-02 & $0 \%$ \\
\hline U-238 & 4.77E+01 & 8.53E+01 & 4.03E+01 & $3.44 E+03$ & $0 \%$ & 0.001 & $3.44 E+00$ & $0 \%$ \\
\hline Np-237 & 7.47E-03 & 1.34E-02 & $3.45 E+05$ & $4.61 E+03$ & $0 \%$ & 0.001 & $4.61 E+00$ & $0 \%$ \\
\hline Pu-236 & $9.38 E-08$ & $1.68 \mathrm{E}-07$ & $8.53 E+10$ & $1.43 E+04$ & $0 \%$ & 0.001 & $1.43 E+01$ & $0 \%$ \\
\hline Pu-238 & 6.68E-05 & 1.20E-04 & $7.88 E+09$ & $9.42 E+05$ & $0 \%$ & 0.001 & $9.42 E+02$ & $0 \%$ \\
\hline Pu-239 & 5.59E-01 & $1.00 E+\infty 0$ & $3.16 E+07$ & $3.16 E+07$ & $4 \%$ & 0.001 & $3.16 E+04$ & $3 \%$ \\
\hline Pu-240 & 3.41E-02 & 6.11E-02 & $1.16 E+08$ & $7.08 E+06$ & $1 \%$ & 0.001 & $7.08 E+03$ & $1 \%$ \\
\hline Pu-241 & 3.24E-03 & 5.79E-03 & $1.03 E+09$ & 5.97E+06 & $1 \%$ & 0.001 & 5.97E +03 & $1 \%$ \\
\hline$P u-242$ & $1.49 E-04$ & 2.67E-04 & $1.89 E+06$ & $5.05 E+02$ & $0 \%$ & 0.001 & 5.05E-01 & $0 \%$ \\
\hline Am-241 & 4.56E-02 & 8.16E-02 & $1.79 E+09$ & $1.46 E+08$ & $17 \%$ & 0.001 & $1.46 E+05$ & $13 \%$ \\
\hline$\overline{A m-242 m}$ & 2.02E-05 & 3.62E-05 & 4.96E+09 & $1.80 E+05$ & $0 \%$ & 0.001 & $1.80 E+02$ & $0 \%$ \\
\hline Am-243 & 2.29E-06 & 4.10E-06 & $1.04 E+08$ & 4.27E+02 & $0 \%$ & 0.001 & 4.27E-01 & $0 \%$ \\
\hline $\mathrm{Cm}-242$ & $1.09 E-07$ & 1.94E-07 & $5.62 E+10$ & $1.09 E+04$ & $0 \%$ & 0.001 & $1.09 E+01$ & $0 \%$ \\
\hline$C m-243$ & 2.20E-07 & $3.94 E-07$ & $1.81 E+10$ & $7.13 E+03$ & $0 \%$ & 0.001 & $7.13 E+\infty$ & $0 \%$ \\
\hline Cm-244 & 3.06E-06 & 5.47E-06 & 2.18E+10 & $1.19 E+05$ & $0 \%$ & 0.001 & $1.19 E+02$ & $0 \%$ \\
\hline Cm-245 & 1.37E-10 & 2.45E-10 & 9.27E+07 & 2.27E-02 & $0 \%$ & 0.001 & 2.27E-05 & $0 \%$ \\
\hline$C m-246$ & $1.41 E-12$ & 2.53E-12 & $1.65 E+08$ & 4.18E-04 & $0 \%$ & 0.001 & 4.18E-07 & $0 \%$ \\
\hline $\mathrm{Cm}-247$ & $1.14 E-15$ & 2.04E-15 & $4.55 E+04$ & $9.28 \mathrm{E}-11$ & $0 \%$ & 0.001 & $9.28 E-14$ & $0 \%$ \\
\hline $\mathrm{Cm}-248$ & $5.02 E-18$ & 8.98E-18 & $8.08 E+06$ & 7.26E-11 & $0 \%$ & 0.001 & 7.26E-14 & $0 \%$ \\
\hline Bk-249 & 2.84E-24 & 5.07E-24 & 2.17E+09 & $1.10 \mathrm{E}-14$ & $0 \%$ & 0.001 & 1.10E-17 & $0 \%$ \\
\hline \multirow[t]{2}{*}{ Cf-248 } & 1.56E-22 & 2.78E-22 & $2.20 \mathrm{E}+08$ & 6.13E-13 & $0 \%$ & 0.001 & 6.13E-16 & $0 \%$ \\
\hline & & & & $8.49 E+08$ & & & $1.14 E+06$ & \\
\hline
\end{tabular}




\section{PUREX RADIONUCLIDE INHALATION DOSE CONTRIBUTION}

Mark 31B Fuel Assembly

15 years

Normalized Yolatile Conditions

Isotope Isotopic Content Sludge Content Dose Conversion Dose Percent Release Dose Percent (g/assembly) (g/g Pu-239) (ICRP-30, rem/g) (rem/g Pu-239) Dose Fraction Values (rem/g Pu-239) Dose

\begin{tabular}{|c|c|c|c|c|c|c|c|c|}
\hline$\overline{\mathrm{H}-3}$ & 1.76E-06 & $0.00 E+00$ & $9.22 E+05$ & $0.00 E+00$ & $0 \%$ & 1 & $0.00 E+\infty 0$ & $0 \%$ \\
\hline $\mathrm{Cr}-51$ & 2.89E-63 & $4.91 E-63$ & $2.40 E+07$ & $1.18 E-55$ & $0 \%$ & 0.001 & $1.18 E-58$ & $0 \%$ \\
\hline Co-60 & 4.08E-04 & 7.30E-0.4 & $1.70 E+08$ & $1.24 E+05$ & $0 \%$ & 0.001 & $1.24 E+02$ & $0 \%$ \\
\hline Se-79 & 1.84E-02 & 2.96E-02 & $6.20 E+02$ & $1.83 E+01$ & $0 \%$ & 0.01 & 1.83E-01 & $0 \%$ \\
\hline $\mathrm{Ab}-87$ & 7.83E-01 & 7.01E-02 & 2.87E-04 & 2.01E-05 & $0 \%$ & 0.001 & $2.01 E-08$ & $0 \%$ \\
\hline Sr-89 & 2.23E-32 & $4.00 E-32$ & $1.07 E+09$ & $4.28 E-23$ & $0 \%$ & 0.001 & 4.28E-26 & $0 \%$ \\
\hline Sr-90 & $1.28 E+00$ & $2.28 E+\infty 0$ & $1.81 \mathrm{E}+08$ & $4.13 E+08$ & $68 \%$ & 0.001 & 4.13E+05 & $67 \%$ \\
\hline$Y-90$ & $3.32 E-04$ & 5.94E-04 & 4.46E +09 & 2.65E+06 & $0 \%$ & 0.001 & $2.65 E+03$ & $0 \%$ \\
\hline$Y-91$ & 1.11E-28 & 1.99E-28 & $1.08 E+09$ & 2.15E-19 & $0 \%$ & 0.001 & 2.15E-22 & $0 \%$ \\
\hline $\mathrm{Zr}-93$ & $2.28 E+00$ & 4.07E+00 & $8.04 E+02$ & $3.28 E+03$ & $0 \%$ & 0.001 & $3.28 E+00$ & $0 \%$ \\
\hline$Z r-95$ & 1.35E-25 & 2.42E-25 & $3.86 E+08$ & 9.34E-17 & $0 \%$ & 0.001 & 9.34E-20 & $0 \%$ \\
\hline Nb-95 & $1.56 \mathrm{E}-25$ & 2.80E-25 & $1.76 E+08$ & 4.92E-17 & $0 \%$ & 0.001 & 4.92E-20 & $0 \%$ \\
\hline $\mathrm{Nb}-95 \mathrm{~m}$ & 9.47E-29 & $1.69 E-28$ & $8.08 E+08$ & 1.37E-19 & $0 \%$ & 0.001 & 1.37E-22 & $0 \%$ \\
\hline Tc-99 & $2.65 \mathrm{E}+00$ & $0.00 E+\infty 0$ & 1.27E+02 & $0.00 E+\infty 0$ & $0 \%$ & 0.001 & $0.00 E+00$ & $0 \%$ \\
\hline Ru-103 & $3.35 E-42$ & $3.00 E-42$ & $2.51 E+08$ & 7.52E-34 & $0 \%$ & 0.01 & 7.52E-36 & $0 \%$ \\
\hline Ru-106 & 2.51E-05 & 2.24E-05 & $1.47 E+09$ & $3.30 E+04$ & $0 \%$ & 0.01 & $3.30 E+02$ & $0 \%$ \\
\hline Pd-107 & 5.12E-01 & $9.16 E-01$ & $6.68 E+00$ & $6.12 E+\infty 0$ & $0 \%$ & 0.001 & $6.12 E-03$ & $0 \%$ \\
\hline $\mathrm{Cd}-115 \mathrm{~m}$ & $3.10 E-40$ & $5.28 E-40$ & $1.65 E+09$ & 8.71E-31 & $0 \%$ & 0.001 & 8.71E-34 & $0 \%$ \\
\hline $\ln -115$ & 2.60E-02 & 4.42E-02 & 1.77E-05 & 7.82E-07 & $0 \%$ & 0.001 & 7.82E-10 & $0 \%$ \\
\hline $\mathrm{Sn-121m}$ & 7.36E-06 & 1.26E-05 & $3.48 E+05$ & $4.40 E+00$ & $0 \%$ & 0.001 & 4.40E-03 & $0 \%$ \\
\hline $\mathrm{Sn}-123$ & 2.41E-15 & 4.15E-15 & 2.47E+08 & 1.02E-06 & $0 \%$ & 0.001 & $1.02 E-09$ & $0 \%$ \\
\hline Sn-126 & 7.99E-02 & 1.37E-01 & $2.44 E+03$ & $3.35 E+02$ & $0 \%$ & 0.001 & 3.35E-01 & $0 \%$ \\
\hline Sb-124 & 1.94E-32 & 3.45E-32 & $3.67 E+08$ & 1.27E-23 & $0 \%$ & 0.001 & 1.27E-26 & $0 \%$ \\
\hline$S b-125$ & $1.22 \mathrm{E}-03$ & $2.18 E-03$ & $1.01 E+07$ & $2.20 E+04$ & $0 \%$ & 0.001 & $2.20 E+01$ & $0 \%$ \\
\hline$S b-126$ & $3.80 \mathrm{E}-10$ & $6.53 \mathrm{E}-10$ & $8.36 \mathrm{E}+08$ & $5.46 E-01$ & $0 \%$ & 0.001 & 5.46E-04 & $0 \%$ \\
\hline$T_{\theta-127 m}$ & $1.83 E-17$ & 3.28E-17 & $1.79 E+08$ & 5.87E-09 & $0 \%$ & 0.01 & 5.87E-11 & $0 \%$ \\
\hline To-129m & 4.16E-51 & 7.44E-51 & $6.03 E+08$ & $4.49 E-42$ & $0 \%$ & 0.01 & $4.49 E-44$ & $0 \%$ \\
\hline $\mid-129$ & 5.60E-01 & $0.00 E+\infty 0$ & $3.18 E+01$ & $0.00 E+00$ & $0 \%$ & 0.5 & $0.00 E+\infty 0$ & $0 \%$ \\
\hline Cs-134 & 1.73E-04 & $1.55 \mathrm{E}-05$ & $6.08 E+07$ & $9.43 E+02$ & $0 \%$ & 0.01 & $9.43 E+\infty 0$ & $0 \%$ \\
\hline Cs-135 & 3.19E-01 & 2.86E-02 & $5.18 E+00$ & $1.48 E-01$ & $0 \%$ & 0.01 & $1.48 E-03$ & $0 \%$ \\
\hline C8-137 & $2.70 E+\infty 0$ & 2.42E-01 & 2.77E+06 & $6.69 E+05$ & $0 \%$ & 0.01 & $6.69 E+03$ & $1 \%$ \\
\hline$\overline{C \theta-141}$ & 4.82E-51 & $8.62 E-51$ & $2.42 E+08$ & $2.09 E-42$ & $0 \%$ & 0.001 & $2.09 E-45$ & $0 \%$ \\
\hline Co-144 & 4.73E-06 & 8.46E-06 & $1.12 E+09$ & $9.47 E+03$ & $0 \%$ & 0.001 & $9.47 E+00$ & $0 \%$ \\
\hline Nd-144 & $3.20 E+00$ & $5.73 E+00$ & $0.00 E+\infty 0$ & $0.00 E+00$ & $0 \%$ & 0.001 & $0.00 E+00$ & $0 \%$ \\
\hline Pm-147 & $2.58 E-02$ & 4.62E-02 & $3.15 E+07$ & $1.46 \mathrm{E}+06$ & $0 \%$ & 0.001 & $1.46 E+03$ & $0 \%$ \\
\hline$P m-148$ & $8.72 E-45$ & $1.56 E-44$ & $1.64 E+09$ & 2.56E-35 & $0 \%$ & 0.001 & $2.56 E-38$ & $0 \%$ \\
\hline Pm-148m & $9.72 E-43$ & $1.74 E-42$ & $2.14 E+08$ & 3.72E-34 & $0 \%$ & 0.001 & 3.72E-37 & $0 \%$ \\
\hline $\mathrm{Sm}-147$ & $1.34 E+\infty$ & $2.39 E+00$ & $1.65 \mathrm{E}+00$ & $3.95 E+00$ & $0 \%$ & 0.001 & $3.95 E-03$ & $0 \%$ \\
\hline Sm-151 & $1.63 E-01$ & 2.92E-01 & $7.63 E+05$ & $2.23 E+0.5$ & $0 \%$ & 0.001 & $2.23 E+02$ & $0 \%$ \\
\hline Eu-152 & 2.21E-04 & 3.96E-04 & $3.81 E+07$ & $1.51 E+04$ & $0 \%$ & 0.001 & $1.51 E+01$ & $0 \%$ \\
\hline Eu-154 & $3.05 E-03$ & 5.46E-03 & $6.86 \mathrm{E}+07$ & $3.75 E+05$ & $0 \%$ & 0.001 & $3.75 E+02$ & $0 \%$ \\
\hline Eu-155 & $4.40 E-03$ & 7.87E-03 & $1.81 E+07$ & $1.42 E+05$ & $0 \%$ & 0.001 & $1.42 E+02$ & $0 \%$ \\
\hline Gd-152 & 1.06E-05 & $1.89 E-0.5$ & $5.15 E-03$ & 9.73E-08 & $0 \%$ & 0.001 & 9.73E-11 & $0 \%$ \\
\hline $\mathrm{Tb}-160$ & 7.52E-28 & 1.34E-27 & $2.48 E+08$ & $3.34 E-19$ & $0 \%$ & 0.001 & 3.34E-22 & $0 \%$ \\
\hline $\mathrm{Ra}-224$ & $3.29 E-11$ & 5.89E-11 & 4.67E+11 & $2.75 E+01$ & $0 \%$ & 0.001 & 2.75E-02 & $0 \%$ \\
\hline Th-228 & $6.40 \mathrm{E}-09$ & 1.14E-08 & $2.54 E+11$ & $2.91 E+03$ & $0 \%$ & 0.001 & $2.91 E+\infty 0$ & $0 \%$ \\
\hline U-232 & 2.44E-07 & 4.37E-07 & $1.48 E+10$ & $6.46 E+03$ & $0 \%$ & 0.001 & $6.46 E+\infty 0$ & $0 \%$ \\
\hline U-234 & $9.70 E-18$ & $1.74 E-17$ & $8.08 E+05$ & 1.40E-11 & $0 \%$ & 0.001 & $1.40 \mathrm{E}-14$ & $0 \%$ \\
\hline U-235 & 7.95E-02 & 1.42E-01 & $2.59 E+02$ & $3.68 E+01$ & $0 \%$ & 0.001 & $3.68 E-02$ & $0 \%$ \\
\hline U-236 & 2.75E-03 & 4.93E-03 & $7.76 E+03$ & $3.82 E+01$ & $0 \%$ & 0.001 & 3.82E-02 & $0 \%$ \\
\hline U-238 & 4.77E+01 & $8.54 E+01$ & $4.03 E+01$ & $3.44 E+03$ & $0 \%$ & 0.001 & $3.44 E+\infty 0$ & $0 \%$ \\
\hline Np-237 & 7.47E-03 & $1.34 E-02$ & $3.45 E+05$ & $4.61 E+03$ & $0 \%$ & 0.001 & $4.61 E+\infty 0$ & $0 \%$ \\
\hline Pu-236 & 8.25E-09 & $1.48 E-08$ & 8.53E+10 & $1.26 E+03$ & $0 \%$ & 0.001 & $1.26 E+\infty$ & $0 \%$ \\
\hline Pu-238 & $6.25 E-05$ & $1.12 E-04$ & $7.88 E+09$ & $8.81 E+05$ & $0 \%$ & 0.001 & $8.81 E+02$ & $0 \%$ \\
\hline Pu-2a? & $5.59 E-01$ & $1.00 E+\infty$ & $3.16 E+07$ & $3.16 E+07$ & $5 \%$ & 0.001 & $3.16 E+04$ & $5 \%$ \\
\hline
\end{tabular}




\section{PUREX RADIONUCLIDE INHALATION DOSE CONTRIBUTION}

Mark 31B Fud Assembiy

15 yoars

Normalized Volatileconditions

Isotope Isotopic Content Sludge Content Dose Conversion Dose Percent (g/assombly)
Dose (g/g Pu-239) (ICRP-30, rem/g) (rem/g Pur-239)
Rolease Percent

\begin{tabular}{lll}
\hline Pu-240 & $3.41 E-02$ & $6.10 E-02$ \\
Pu-241 & $2.02 E-03$ & $3.62 E-03$ \\
Pu-242 & $1.49 E-04$ & $2.67 E-04$ \\
Am-241 & $4.61 E-02$ & $8.25 E-02$ \\
Am-242m & $1.93 E-05$ & $3.46 E-05$ \\
Am-243 & $2.29 E-06$ & $4.10 E-06$ \\
Cm-242 & $4.68 E-08$ & $8.38 E-08$ \\
Cm-243 & $1.73 E-07$ & $3.09 E-07$ \\
Cm-244 & $2.09 E-08$ & $3.73 E-08$ \\
Cm-245 & $1.37 E-10$ & $2.45 E-10$ \\
Cm-246 & $1.41 E-12$ & $2.53 E-12$ \\
Cm-247 & $1.14 E-15$ & $2.04 E-15$ \\
Cm-248 & $5.02 E-18$ & $8.99 E-18$ \\
Bk-249 & $8.93 E-28$ & $1.60 E-27$ \\
Cf-249 & $1.55 E-22$ & $2.78 E-22$
\end{tabular}

$1.16 E+08$
$1.03 E+09$
$1.89 E+06$
$1.79 E+09$
$4.96 E+09$
$1.04 E+08$
$5.62 E+10$
$1.81 E+10$
$2.18 E+10$
$9.27 E+07$
$1.65 E+08$
$4.55 E+04$
$8.08 E+06$
$2.17 E+09$
$2.20 E+09$

Evaporative Conditions.

\begin{tabular}{l}
$7.08 E+06$ \\
$3.72 E+06$ \\
$5.05 E+02$ \\
$1.48 E+08$ \\
$1.72 E+05$ \\
$4.26 E+02$ \\
$4.71 E+03$ \\
$5.59 E+03$ \\
$8.14 E+02$ \\
$2.27 E-02$ \\
$4.17 E-04$ \\
$9.28 E-11$ \\
$7.26 E-11$ \\
$3.47 E-18$ \\
$6.12 E-13$ \\
\hline
\end{tabular}

$\begin{array}{ll}\frac{1 \%}{1 \%} & 0.001 \\ \frac{0 \%}{0 \%} & 0.001 \\ 24 \% & 0.001 \\ 0 \% & 0.001 \\ 0 \% & 0.001 \\ 0 \% & 0.001 \\ 0 \% & 0.001 \\ 0 \% & 0.001 \\ 0 \% & 0.001 \\ 0 \% & 0.001 \\ 0 \% & 0.001 \\ 0 \% & 0.001 \\ 0 \% & 0.001 \\ 0 \% & 0.001 \\ 0.001\end{array}$
$6.10 E+08$

\begin{tabular}{lc}
\hline $7.08 E+03$ & $1 \%$ \\
$3.72 E+03$ & $1 \%$ \\
$5.05 E-01$ & $0 \%$ \\
$1.48 E+05$ & $\frac{24 \%}{0 \%}$ \\
$1.72 E+02$ & $0 \%$ \\
$4.26 E-01$ & $0 \%$ \\
$4.71 E+00$ & $0 \%$ \\
$5.59 E+00$ & $0 \%$ \\
$8.14 E-01$ & $0 \%$ \\
$2.27 E-05$ & $0 \%$ \\
$4.17 E-07$ & $0 \%$ \\
$9.28 E-14$ & $0 \%$ \\
$7.26 E-14$ & $0 \%$ \\
$3.47 E-21$ & $0 \%$ \\
$6.12 E-16$ & $0 \%$
\end{tabular}




\section{PUAEX RADIONUCUDE INGESTION DOSE CONTRIBUTION}

Mark 31A Fuol Aseambly

5 yoars

Normalized

Isotope Isotopic Content Sludge Content Dose Comversion Dose Percent (g/assembly) ( $g / g$ Pu-239) (ICRP-30, rem/g) (rem/g Pu-239) Dose

\begin{tabular}{|c|c|c|c|c|c|}
\hline H-3 & 3.64E-05 & $0.00 E+00$ & $6.11 E+05$ & $0.00 E+00$ & $0 \%$ \\
\hline Cr-51 & 3.03E-23 & $3.61 E-23$ & $1.20 E+07$ & 4.33E-16 & $0 \%$ \\
\hline Co-60 & $4.50 \mathrm{E}-03$ & 5.65E-03 & 2.94E+07 & $1.66 E+05$ & $0 \%$ \\
\hline Se-79 & 2.51E-02 & 2.84E-02 & $5.56 E+02$ & $1.58 E+01$ & $0 \%$ \\
\hline$R b-87$ & $1.04 E+00$ & 6.54E-02 & 4.17E-04 & 2.73E-05 & $0 \%$ \\
\hline Sr-89 & 3.87E-11 & 4.86E-11 & $2.53 E+08$ & $1.23 E-02$ & $0 \%$ \\
\hline Sr-en & $2.16 E+00$ & $2.72 E+\infty$ & $1.81 E+07$ & $4.92 E+07$ & $79 \%$ \\
\hline$Y-90$ & $5.63 E-04$ & 7.07E-04 & $5.43 E+09$ & $3.84 E_{+}+06$ & $6 \%$ \\
\hline$Y-91$ & 8.07E-10 & $1.01 E-09$ & $2.18 E+08$ & 2.21E-01 & $0 \%$ \\
\hline $\mathrm{Zr}-93$ & $3.05 E+00$ & $3.83 E+\infty 0$ & $4.02 E+\infty$ & $1.54 E+01$ & $0 \%$ \\
\hline $\mathrm{Zr}-95$ & $1.06 \mathrm{E}-08$ & $1.33 E-08$ & $7.30 E+07$ & 9.68E-01 & $0 \%$ \\
\hline $\mathrm{Nb}-95$ & $1.22 E-08$ & $1.53 \mathrm{E}-08$ & 8.63E+07 & $1.32 E+00$ & $0 \%$ \\
\hline $\mathrm{Nb}-95 \mathrm{~m}$ & $7.39 E-12$ & $9.28 E-12$ & $7.35 E+08$ & $6.82 E-03$ & $0 \%$ \\
\hline Tc-99 & $3.59 E+00$ & $0.00 E+\infty 0$ & $2.20 E+01$ & $0.00 E+00$ & $0 \%$ \\
\hline$R u-103$ & $2.50 E-14$ & $1.57 \mathrm{E}-14$ & $8.70 E+07$ & 1.37E-06 & $0 \%$ \\
\hline$R u-106$ & $3.38 E-02$ & 2.12E-02 & $7.03 E+07$ & $1.49 E+06$ & $2 \%$ \\
\hline Pd-107 & 7.27E-01 & 9.13E-01 & 7.20E-02 & 6.57E-02 & $\overline{0 \%}$ \\
\hline $\operatorname{cod}-115 m$ & $2.14 E-15$ & $2.55 E-15$ & $3.81 E+08$ & $9.73 E-07$ & $0 \%$ \\
\hline $\ln -115$ & 4.24E-02 & 5.06E-02 & 7.28E-07 & 3.69E-08 & $0 \%$ \\
\hline $\mathrm{sn}-121 \mathrm{~m}$ & 1.17E-05 & 1.41E-05 & $5.08 E+04$ & 7.17E-01 & $0 \%$ \\
\hline $5 n-123$ & $1.25 E-06$ & $1.51 E-06$ & $6.33 E+07$ & $9.57 E+01$ & $0 \%$ \\
\hline Sn-125 & 1.10E-59 & 1.32E-59 & $1.14 E+07$ & 1.51E-52 & $0 \%$ \\
\hline Sn-126 & $1.20 E-01$ & $1.45 E-01$ & $4.82 E+02$ & 6.97E+01 & $0 \%$ \\
\hline Sb-124 & $6.08 E-14$ & $7.59 E-14$ & $1.63 E+08$ & $1.24 E-05$ & $0 \%$ \\
\hline Sb-125 & 2.39E-02 & $2.98 E-02$ & $2.69 E+06$ & $8.03 E+04$ & $0 \%$ \\
\hline$S b-126$ & 5.70E-10 & $6.87 E-10$ & $6.10 E+06$ & 4.19E-03 & $0 \%$ \\
\hline $\mathrm{T} \theta-127 \mathrm{~m}$ & $3.16 E-07$ & 3.96E-07 & $7.45 E+07$ & $2.95 E+01$ & $0 \%$ \\
\hline To-129m & 4.41E-18 & $5.54 E-18$ & $2.98 E+08$ & 1.65E-09 & $0 \%$ \\
\hline $1-129$ & 7.85E-01 & $0.00 E+00$ & $4.94 E+01$ & $0.00 E+00$ & $0 \%$ \\
\hline $\mid-131$ & 2.81E-69 & $0.00 E+00$ & $6.57 E+09$ & $0.00 E+00$ & $0 \%$ \\
\hline Cs-134 & $6.39 E-03$ & $4.01 E-04$ & $9.58 E+07$ & $3.84 E+04$ & $0 \%$ \\
\hline Cs-135 & 5.91E-01 & $3.71 E-02$ & 8.17E+00 & $3.03 E-01$ & $0 \%$ \\
\hline Cs-136 & $6.85 E-45$ & $4.30 E-46$ & $8.03 E+08$ & $3.45 E-37$ & $0 \%$ \\
\hline C8-137 & $4.59 E+00$ & $2.88 E-01$ & $4.33 E+06$ & $1.25 E+06$ & $2 \%$ \\
\hline $\mathrm{Ba}-140$ & $1.86 E-43$ & 2.33E-43 & $6.13 E+08$ & $1.43 E-34$ & $0 \%$ \\
\hline La-140 & $2.80 \mathrm{E}-44$ & $3.52 E-44$ & $4.29 E_{+09}$ & 1.51E-34 & $0 \%$ \\
\hline Co-141 & 3.72E-17 & 4.67E-17 & 7.41E+07 & 3.46E-09 & $0 \%$ \\
\hline Ce-144 & 4.60E-02 & $5.78 E-02$ & $6.38 E+07$ & $3.69 E+06$ & $6 \%$ \\
\hline$P_{t-143}$ & $6.19 E-41$ & 7.77E-41 & $3.03 E+08$ & 2.35E-32 & $0 \%$ \\
\hline Nd-144 & $4.25 E+\infty 0$ & $5.34 E+00$ & $0.00 E+\infty 0$ & $0.00 E+00$ & $0 \%$ \\
\hline Nd-147 & $5.90 E-51$ & 7.41E-51 & $3.16 E+08$ & $2.34 E-42$ & $0 \%$ \\
\hline Pm-147 & 4.82E-01 & $6.05 E-01$ & $8.81 E+05$ & 5.33E+05 & 18 \\
\hline$P m-148$ & 6.65E-18 & $8.35 E-18$ & $1.56 E+09$ & $1.30 \mathrm{E}-08$ & $0 \%$ \\
\hline Pm-148m & 7.41E-16 & 9.31E-16 & $1.50 E+08$ & $1.40 \mathrm{E}-07$ & $0 \%$ \\
\hline Sm-147 & $1.33 E+\infty 0$ & $1.68 E+00$ & $4.19 E-03$ & 7.02E-03 & $0 \%$ \\
\hline Sm-151 & 2.59E-01 & $3.25 E-01$ & $8.95 E+03$ & $2.91 E+03$ & $0 \%$ \\
\hline Eu-152 & 4.85E-04 & $6.09 E-04$ & $1.04 E+06$ & $6.33 E+02$ & $0 \%$ \\
\hline Eu-154 & $1.03 E-02$ & 1.30E-02 & 2.40E+06 & $3.11 E+04$ & $0 \%$ \\
\hline Eu-155 & 2.76E-02 & $3.46 E-02$ & $6.05 E+05$ & $2.09 E+04$ & $0 \%$ \\
\hline Eu-156 & $4.00 E-38$ & $5.02 E-38$ & $4.79 E+08$ & $2.41 E-29$ & $0 \%$ \\
\hline Gd-152 & $1.75 E-05$ & 2.20E-05 & $3.22 E-06$ & $7.08 E-11$ & $0 \%$ \\
\hline Tb-160 & 2.12E-12 & 2.66E-12 & $7.23 E+07$ & 1.93E-04 & $0 \%$ \\
\hline Ra-224 & $4.31 E-15$ & 5.42E-15 & $5.31 E+10$ & $2.88 E-04$ & $0 \%$ \\
\hline Th-228 & 8.36E-13 & $1.05 E-12$ & $3.11 E+08$ & 3.27E-0. & $0 \%$ \\
\hline
\end{tabular}




\section{PUREX RADIONUCLIDE INGESTION DOSE CONTRIBUTION}

\begin{tabular}{|c|c|c|c|c|c|}
\hline Isotope & $\begin{array}{l}\text { Isotopic Content } \\
\text { (g/assembly) }\end{array}$ & $\begin{array}{l}\text { Normalized } \\
\text { Sudge Content } \\
\text { (g/g Pu-239) }\end{array}$ & $\begin{array}{l}\text { Dose Conversion } \\
\text { (ICRP-30, rem/g) }\end{array}$ & $\begin{array}{c}\text { Dose } \\
\text { rem/g Pu-239) }\end{array}$ & $\begin{array}{c}\text { Percent } \\
\text { Dose }\end{array}$ \\
\hline U-232 & $1.56 \mathrm{E}-11$ & 1.क्5E-11 & $2.86 E+07$ & $5.59 E-04$ & $0 \%$ \\
\hline$U-234$ & 1.42E- 17 & $1.79 \mathrm{E}-17$ & $1.62 E+03$ & 2.90E-14 & $0 \%$ \\
\hline U-235 & $1.20 E-01$ & 1.51E-01 & 5.41E-01 & 8.15E-02 & $0 \%$ \\
\hline U-236 & $3.74 E-03$ & 4.69E-03 & $1 . œ E+01$ & $7.60 \mathrm{E}-02$ & $0 \%$ \\
\hline U-238 & $7.03 E+01$ & $8.82 E+01$ & 7.73E-02 & $6.82 E+\infty$ & $0 \%$ \\
\hline Np-237 & $1.21 E-02$ & $1.52 E-02$ & $2.75 E+03$ & 4.19E+01 & $0 \%$ \\
\hline Pu-236 & 8.01E-12 & $1.01 E-11$ & $6.93 E+08$ & $6.97 E-03$ & $0 \%$ \\
\hline Pu-238 & 1.26E-04 & $1.58 E-04$ & $6.51 E+07$ & $1.03 E+04$ & $0 \%$ \\
\hline Pu-239 & 7.96E-01 & $1.00 E+00$ & $2.67 E+05$ & $2.67 E+05$ & $0 \%$ \\
\hline Pu-240 & $4.59 E-02$ & $5.76 \mathrm{E}-02$ & $9.75 E+05$ & $5.61 E+04$ & $0 \%$ \\
\hline Pu-241 & 5.12E-03 & $6.43 E-03$ & $8.86 E+06$ & $5.70 E+04$ & $0 \%$ \\
\hline Pu-242 & 2.13E-04 & 2.68E-04 & $1.61 E+04$ & $4.31 E+00$ & $0 \%$ \\
\hline$A m-241$ & 7.34E-02 & $9.21 E-02$ & $1.54 E+07$ & $1.42 \mathrm{E}+06$ & $2 \%$ \\
\hline Am-242m & $4.00 E-05$ & 5.03E-05 & $4.08 E+07$ & $2.05 E+03$ & $\overline{0 \%}$ \\
\hline Am-243 & 4.05E-06 & $5.08 E-06$ & 8.87E+05 & $4.56 E+00$ & $0 \%$ \\
\hline Cm-242 & 2.09E-07 & 2.63E-07 & $3.64 E+08$ & $9.56 \mathrm{E}+01$ & $0 \%$ \\
\hline $\mathrm{Cm}-243$ & 4.89E-07 & $6.14 E-07$ & $1.50 E+08$ & 9.21E+01 & $0 \%$ \\
\hline $\mathrm{Cm}-244$ & $6.53 E-08$ & 8.20E-08 & $1.86 E+08$ & $1.52 E+01$ & $0 \%$ \\
\hline Cm-245 & 3.72E-10 & 4.67E-10 & $7.73 E+05$ & 3.61E-04 & $0 \%$ \\
\hline $\mathrm{Cm}-246$ & 3.51E-12 & 4.41E-12 & $1.38 E+06$ & $6.09 E-06$ & $0 \%$ \\
\hline $\mathrm{Cm}-247$ & $3.66 \mathrm{E}-15$ & 4.60E-15 & $3.80 \mathrm{E}+02$ & 1.75E-12 & $0 \%$ \\
\hline $\mathrm{Cm}-248$ & $1.93 \mathrm{E}-17$ & 2.42E-17 & $6.80 E+04$ & 1.64E-12 & $0 \%$ \\
\hline BK-249 & 1.06E-23 & 1.33E-23 & $1.00 E+07$ & 1.33E-16 & $0 \%$ \\
\hline Cf-249 & $5.80 E-22$ & 7.28E-22 & $1.84 E+07$ & 1.34E-14 & $0 \%$ \\
\hline
\end{tabular}


PUREX RADKONUCLIDE INGESTION DOSE CONTRIBUTION

Mark 31A Fuet Assembly

15 years

Normalized

Isotope Isotopic Content Sludge Content Dose Corversion Dose Percent (g/assembly) ( $g / g$ Pu-239) (ICRP-30, rem/g) (rem/g Pu-239) Dose

\begin{tabular}{|c|c|c|c|c|c|}
\hline$\overline{H 3}$ & $2.09 E-05$ & $0.00 E+00$ & $6.11 E+05$ & $0.00 \mathrm{E}+00$ & $0 \%$ \\
\hline$C_{r}-51$ & $8.51 E-63$ & $1.02 E-62$ & $1.20 \mathrm{E}+07$ & 1.22E-55 & $0 \%$ \\
\hline Co-60 & $1.20 E-03$ & 1.51E-03 & $2.94 E+07$ & $4.45 E+04$ & $0 \%$ \\
\hline Se-79 & 2.51E-02 & 2.84E-02 & $5.56 E+02$ & $1.58 E+01$ & $0 \%$ \\
\hline $\mathrm{Rb}-87$ & $1.04 E+00$ & $6.54 E-02$ & 4.17E-04 & 2.73E-05 & $0 \%$ \\
\hline Sr-89 & 2.78E-32 & $3.49 E-32$ & $2.53 E+08$ & $8.82 E-24$ & $0 \%$ \\
\hline Sr -90 & $1.69 E+00$ & $2.12 E+00$ & $1.81 E+07$ & $3.85 E+07$ & $87 \%$ \\
\hline$Y-90$ & 4.40E-04 & 5.53E-04 & $5.43 E+09$ & $3.00 E+06$ & $7 \%$ \\
\hline$\overline{Y-91}$ & 1.39E-28 & 1.75E-28 & $2.18 E+08$ & 3.81E-20 & $0 \%$ \\
\hline Zr-93 & $3.04 E+00$ & $3.82 E+00$ & $4.02 E+00$ & $1.54 \mathrm{E}+01$ & $0 \%$ \\
\hline Zr-95 & 1.73E- 25 & 2.17E-25 & $7.30 E+07$ & $1.58 E-17$ & $0 \%$ \\
\hline $\mathrm{Nb}-95$ & $1.99 E-25$ & 2.51E-25 & 8.63E+07 & 2.16E-17 & $0 \%$ \\
\hline $\mathrm{Nb}-95 \mathrm{~m}$ & 1.21E-28 & 1.52E-28 & $7.35 E+08$ & 1.12E-19 & $0 \%$ \\
\hline Tc-99 & $3.59 E+00$ & $0.00 E+00$ & $2.20 E+01$ & $0.00 E+\infty 0$ & $0 \%$ \\
\hline$R u-103$ & $4.29 E-42$ & $2.69 E-42$ & $8.70 E+07$ & $2.34 E-34$ & $0 \%$ \\
\hline Ru-106 & 3.54E-05 & 2.23E-05 & $7.03 E+07$ & $1.57 E+03$ & $0 \%$ \\
\hline Pd-107 & 7.27E-01 & 9.13E-01 & 7.20E-02 & 6.58E-02 & $0 \%$ \\
\hline Cd-115m & $4.76 E-40$ & $5.68 E-40$ & $3.81 E+08$ & $2.16 E-31$ & $0 \%$ \\
\hline $\ln -115$ & 4.24E-02 & 5.07E-02 & 7.28E-07 & $3.69 E-08$ & $0 \%$ \\
\hline Sn-121m & $1.02 E-05$ & 1.23E-05 & $5.08 E+04$ & 6.24E-01 & $0 \%$ \\
\hline Sn-123 & 3.76E-15 & 4.54E-15 & $6.33 E+07$ & 2.87E-07 & $0 \%$ \\
\hline Sn-126 & $1.20 E-01$ & $1.45 E-01$ & $4.82 E+02$ & $6.97 E+01$ & $0 \%$ \\
\hline$S b-124$ & 3.31E-32 & 4.13E-32 & $1.63 E+08$ & 6.74E-24 & $0 \%$ \\
\hline Sb-125 & $1.89 E-03$ & 2.36E-03 & 2. $69 E+06$ & $6.34 E+03$ & $0 \%$ \\
\hline Sb-126 & 5.70E-10 & 6.87E-10 & $6.10 E+06$ & 4.19E-03 & $0 \%$ \\
\hline Te-127m & 2.58E-17 & $3.24 E-17$ & 7.45E+07 & 2.41E-09 & $0 \%$ \\
\hline Te-129m & 5.31E-51 & 6.67E-51 & $2.98 E+08$ & $1.99 E-42$ & $0 \%$ \\
\hline $\mid-129$ & 7.85E-01 & $0.00 E+\infty$ & $4.94 E+01$ & $0.00 E+00$ & $0 \%$ \\
\hline Cs-134 & 2.90E-04 & $1.82 E-05$ & $9.58 E+07$ & $1.74 E+03$ & $0 \%$ \\
\hline Cs-135 & 5.91E-01 & 3.71E-02 & $8.17 E+\infty$ & $3.03 E-01$ & $0 \%$ \\
\hline C8-137 & $3.65 E+00$ & $2.29 E-01$ & $4.33 E+06$ & $9.92 E+05$ & $2 \%$ \\
\hline$\overline{C_{\theta-141}}$ & 5.80E-51 & 7.41E-51 & $7.41 E+07$ & $5.49 E-43$ & $\overline{0 \%}$ \\
\hline$C_{\theta-144}$ & 6.26E-06 & 7.87E-06 & $6.38 E+07$ & $5.02 E+02$ & $0 \%$ \\
\hline Nd-144 & $4.30 \mathrm{E}+00$ & $5.40 E+00$ & $0.00 E+00$ & $0.00 E+00$ & $0 \%$ \\
\hline Pm-147 & 3.43E-02 & 4.31E-02 & $8.81 E+05$ & $3.79 E+04$ & $0 \%$ \\
\hline Pm-148 & $1.59 E-44$ & $1.89 E-44$ & $1.56 E+08$ & $3.11 E-35$ & $0 \%$ \\
\hline Pm-148m & $1.77 E-42$ & $2.22 E-42$ & $1.50 E+08$ & 3.33E-34 & $0 \%$ \\
\hline $\mathrm{Sm}-147$ & $1.78 E+00$ & $2.24 E+\infty$ & 4.19E-03 & 9.37E-03 & $0 \%$ \\
\hline Sm-151 & $2.40 E-01$ & $3.02 E-01$ & $8.95 E+03$ & $2.70 E+03$ & $0 \%$ \\
\hline Eu-152 & 2.84E-0.4 & 3.57E-04 & $1.04 E+06$ & $3.71 E+02$ & $0 \%$ \\
\hline Eu-154 & 4.61E-03 & $5.79 E-03$ & $2.40 E+06$ & $1.39 E+04$ & $0 \%$ \\
\hline Eu-155 & $6.50 E-03$ & $8.16 E-03$ & $6.05 E+05$ & $4.94 E+03$ & $0 \%$ \\
\hline Gd-152 & 1.75E-05 & $2.20 E-05$ & 3.22E-06 & 7.08E-11 & $0 \%$ \\
\hline $\mathrm{Tb}-160$ & $1.32 E-27$ & 1.65E-27 & $7.23 E+07$ & 1.19E-19 & $0 \%$ \\
\hline Ra-224 & 2.81E-15 & 3.53E-15 & $5.31 E+10$ & 1.87E-04 & $0 \%$ \\
\hline Th-228 & $5.46 E-13$ & 6.86E-13 & $3.11 E+08$ & $2.13 E-04$ & $0 \%$ \\
\hline$U-232$ & 2.08E-11 & 2.62E-11 & $2.86 E+07$ & 7.49E-04 & $0 \%$ \\
\hline$U-234$ & 1.42E-17 & $1.79 \mathrm{E}-17$ & $1.62 E+03$ & 2.90E-14 & $0 \%$ \\
\hline U-235 & $1.20 E-01$ & 1.51E-01 & 5.41E-01 & 8.16E-02 & $0 \%$ \\
\hline U-236 & $3.74 E-03$ & $4.68 E-03$ & $1.62 E+01$ & 7.61E-02 & $0 \%$ \\
\hline$U-238$ & 7.03E+01 & $8.82 E+01$ & 7.73E-02 & $6.82 E+00$ & $0 \%$ \\
\hline Np-237 & $1.21 E-02$ & $1.52 E-02$ & $2.75 E+03$ & $4.18 E+01$ & $0 \%$ \\
\hline Put236 & $7.05 E-13$ & 8.85E-13 & $6.93 E+08$ & 6.13E-04 & $0 \%$ \\
\hline$P u-238$ & $1.18 E-04$ & $1.48 E-04$ & $6.51 E+07$ & $9.65 E+03$ & $0 \%$ \\
\hline Pu-239 & $7.96 E-01$ & $1.00 E+\infty$ & $2.67 E+05$ & 2.67E+0.5 & $1 \%$ \\
\hline
\end{tabular}


PUREX RADIONUCLDE INGESTION DOSE CONTRIBUTION

Mark 31A Fuet Assembly

15 yoars

Normalized

Isotope Isotopic Content Sludge Content Dose Conversion Dose Percent (g/assembly) (g/g Pu-239) (ICRP-30, rem/g) (rem/g Pu-239) Dose

\begin{tabular}{llllll}
\hline PU-240 & $4.58 E-02$ & $5.75 E-02$ & $9.75 E+05$ & $5.61 E+04$ & $0 \%$ \\
Pu-241 & $3.20 E-03$ & $4.01 E-03$ & $8.86 E+06$ & $3.56 E+04$ & $0 \%$ \\
Pu-242 & $2.14 E-04$ & $2.68 E-04$ & $1.61 E+04$ & $4.32 E+00$ & $0 \%$ \\
Am-241 & $7.41 E-02$ & $9.31 E-02$ & $1.54 E+07$ & $1.43 E+06$ & $3 \%$ \\
Am-242m & $3.82 E-05$ & $4.80 E-05$ & $4.08 E+07$ & $1.96 E+03$ & $0 \%$ \\
Am-243 & $4.04 E-06$ & $5.08 E-06$ & $8.97 E+05$ & $4.56 E+00$ & $0 \%$ \\
Cm-242 & $9.26 E-08$ & $1.16 E-07$ & $3.64 E+08$ & $4.23 E+01$ & $0 \%$ \\
Cm-243 & $3.84 E-07$ & $4.82 E-07$ & $1.50 E+08$ & $7.23 E+01$ & $0 \%$ \\
Cm-244 & $4.45 E-08$ & $5.59 E-08$ & $1.86 E+08$ & $1.04 E+01$ & $0 \%$ \\
Cm-245 & $3.72 E-10$ & $4.67 E-10$ & $7.73 E+05$ & $3.61 E-04$ & $0 \%$ \\
Cm-246 & $3.51 E-12$ & $4.41 E-12$ & $1.38 E+06$ & $6.08 E-06$ & $0 \%$ \\
Cm-247 & $3.66 E-15$ & $4.60 E-15$ & $3.80 E+02$ & $1.75 E-12$ & $0 \%$ \\
Cm-248 & $1.93 E-17$ & $2.42 E-17$ & $6.80 E+04$ & $1.64 E-12$ & $0 \%$ \\
Bk-249 & $3.33 E-27$ & $4.18 E-27$ & $1.00 E+07$ & $4.18 E-20$ & $0 \%$ \\
Cf-249 & $5.79 E-22$ & $7.27 E-22$ & $1.84 E+07$ & $1.34 E-14$ & $0 \%$
\end{tabular}


PUREX RADIONUCUDE INGESTION DOSE CONTRIBUTION

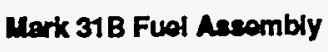

Normalized Isotope Isotopic Content Sludge Content Dose Comversion Dose Percent (g/assembly) (g/gPu-239) (ICRP-30, rem/g) (rem/g Pu-239) Dose

\begin{tabular}{|c|c|c|c|c|c|}
\hline $\mathrm{H3}$ & 3.10E-05 & $0.00 E+00$ & $6.11 E+05$ & $0.00 E+00$ & $0 \%$ \\
\hline $\mathrm{Cr}-51$ & 1.03E-23 & 1.74E-23 & $1.20 E+07$ & $2.09 E-16$ & $0 \%$ \\
\hline Co-60 & 1.53E-03 & 2.73E-03 & 2.94E+07 & $8.03 E+04$ & $0 \%$ \\
\hline Se-79 & 1.84E-02 & 2.96E-02 & $5.56 E+02$ & $1.64 E+01$ & $0 \%$ \\
\hline $\mathrm{Rb}-87$ & 7.83E-01 & 7.01E-02 & 4.17E-04 & $2.92 E-05$ & $0 \%$ \\
\hline Sr-89 & 3.11E-11 & 5.57E-11 & 2.53E+08 & 1.41E-02 & $0 \%$ \\
\hline Sreqe & $1.63 E+00$ & $2.92 E+00$ & $1.81 E+07$ & $5.29 E+07$ & $80 \%$ \\
\hline$Y-90$ & 4.25E-0. & 7.60E-04 & 5.43E+09 & $4.13 E+06$ & $6 \%$ \\
\hline$\overline{Y-91}$ & $6.43 E-10$ & 1.15E-08 & $2.18 E+08$ & 2.51E-01 & $0 \%$ \\
\hline Zr-93 & $2.28 E+00$ & $4.07 E+\infty 0$ & $4.02 E+\infty 0$ & $1.64 E+01$ & $0 \%$ \\
\hline Zr-95 & 8.27E-09 & $1.48 E-08$ & $7.30 E+07$ & $1.08 E+\infty$ & $0 \%$ \\
\hline $\mathrm{Nb}-95$ & $9.56 E-09$ & 1.71E-08 & 8.63E+07 & $1.48 E+00$ & $0 \%$ \\
\hline $\mathrm{Nb}-95 \mathrm{~m}$ & 5.79E-12 & 1.04E-11 & $7.35 E+08$ & 7.61E-03 & $0 \%$ \\
\hline Tc-99 & $2.65 E+\infty 0$ & $0.00 E+\infty$ & $2.20 E+01$ & $0.00 E+00$ & $0 \%$ \\
\hline$R u-103$ & $1.95 E-14$ & $1.75 E-14$ & $8.70 E+07$ & 1.52E-06 & $0 \%$ \\
\hline Bu-106 & 2.39E-02 & 2.14E-02 & 7.03E+07 & $1.50 E+06$ & $2 \%$ \\
\hline Pd-107 & $5.12 E-01$ & 9.16E-01. & 7.20E-02 & $6.59 E-02$ & $0 \%$ \\
\hline $\mathrm{Cd}-115 \mathrm{~m}$ & $1.40 E-15$ & 2.37E-15 & $3.81 E+08$ & 9.04E-07 & $0 \%$ \\
\hline $\ln -115$ & $2.60 E-02$ & 4.41E-02 & $7.28 E-07$ & $3.21 E-08$ & $0 \%$ \\
\hline Sn-121m & 8.45E-06 & 1.45E-0.5 & $5.08 E+04$ & 7.38E-01 & $0 \%$ \\
\hline Sn-123 & 8.05E-07 & $1.38 E-06$ & $6.33 E+07$ & 8.75E+01 & $0 \%$ \\
\hline Sn-125 & $8.85 E-60$ & $1.52 E-59$ & $1.14 E+07$ & $1.73 E-52$ & $0 \%$ \\
\hline Sn-126 & 7.99E-02 & $1.37 E-01$ & $4.82 E+02$ & $6.6 E+01$ & $0 \%$ \\
\hline Sb-124 & 3.56E-14 & 6.33E-14 & $1.63 E+08$ & $1.03 E-05$ & $0 \%$ \\
\hline$S b-125$ & $1.55 E-02$ & 2.76E-02 & $2 . \oplus E+06$ & 7.41E+04 & $0 \%$ \\
\hline$S b-126$ & $3.80 E-10$ & 6.53E-10 & $6.10 E+06$ & $3.98 E-03$ & $0 \%$ \\
\hline Te-127m & $2.24 E-07$ & 4.01E-07 & 7.45E+07 & $2.99 E+01$ & $0 \%$ \\
\hline$T e-129 \mathrm{~m}$ & $3.45 E-18$ & $6.18 E-18$ & $2.88 E+08$ & $1.84 E-09$ & $0 \%$ \\
\hline $1-129$ & $5.60 E-01$ & $0.00 E+00$ & $4.84 E+01$ & $0.00 E+00$ & $0 \%$ \\
\hline$|-13|$ & $2.56 E-69$ & $0.00 E+00$ & 6.57E+09 & $0.00 E+\infty 0$ & $0 \%$ \\
\hline Cs-134 & 5.01E-03 & $4.48 E-04$ & 9.58E+07 & 4.30E+04 & $0 \%$ \\
\hline Cs-135 & 3.19E-01 & 2.86E-02 & 8.17E+00 & 2.33E-01 & $0 \%$ \\
\hline Cs-136 & $5.78 E-45$ & 5.17E-46 & $8.03 E+08$ & 4.15E-37 & $0 \%$ \\
\hline C8-137. & $3.40 E+00$ & 3.04E-01 & $4.33 E+0.6$ & $1.32 E+06$ & $2 \%$ \\
\hline $\mathrm{Ba}-140$ & $1.65 E-43$ & $2.96 E-43$ & $6.13 E+08$ & 1.81E-34 & $0 \%$ \\
\hline La-140 & $2.49 E-44$ & 4.46E-44 & $4.29 E+09$ & 1.91E-34 & $0 \%$ \\
\hline Co-141 & 3.04E-17 & 5.44E-17 & 7.41E+07 & $4.03 E-09$ & $0 \%$ \\
\hline Co-144 & 3.47E-02 & 6.21E-02 & $6.38 E+07$ & $3.96 E+06$ & $6 \%$ \\
\hline Pr-143 & $5.50 E-41$ & $9.83 E-41$ & $3.03 E+08$ & 2.98E-32 & $0 \%$ \\
\hline Nd-144 & $3.17 E+\infty$ & 5.67E+00 & $0.00 E+\infty 0$ & $0.00 E+00$ & $0 \%$ \\
\hline Nd-147 & 5.28E-51 & 9.44E-51 & $3.16 E+08$ & $2.98 E-42$ & $0 \%$ \\
\hline Pm-147 & 3.63E-01 & 6.49E-01 & $8.81 E+05$ & 5.72E+05 & $1 \%$ \\
\hline Pm-148 & 3.66E-18 & $6.54 E-18$ & $1.56 E+09$ & 1.02E-08 & $0 \%$ \\
\hline$P m-148 m$ & 4.08E-16 & $7.29 E-16$ & $1.50 E+08$ & $1.09 E-07$ & $0 \%$ \\
\hline Sm-147 & $1.00 E+\infty 0$ & $1.79 E+00$ & 4.19E-03 & $7.49 E-03$ & $0 \%$ \\
\hline Sm-151 & 1.76E-01 & $3.15 E-01$ & $8.25 E+03$ & $2.82 E+03$ & $0 \%$ \\
\hline Eu-152 & 3.77E-0.4 & 6.75E-0.4 & $1.04 E+06$ & $7.02 E+02$ & $0 \%$ \\
\hline Eu-154 & 6.84E-03 & 1.22E-02 & 2.40E+06 & $2.94 E+04$ & $0 \%$ \\
\hline Eu-155 & $1.86 E-02$ & 3.34E-02 & $6.05 E+05$ & $2.02 E+04$ & $0 \%$ \\
\hline Eu-156 & 3.32E-38 & $5.94 E-38$ & $4.79 E+08$ & 2.85E-29 & $0 \%$ \\
\hline Gd-152 & $1.06 E-05$ & $1.89 E-05$ & 3.22E-06 & $6.08 E-11$ & $0 \%$ \\
\hline Tb-160 & $1.21 E-12$ & 2.17E-12 & 7.23E+07 & 1.57E-0.4 & $0 \%$ \\
\hline Ra-224 & $5.05 E-11$ & $9.04 E-11$ & $5.31 E+10$ & $4.80 E+00$ & $0 \%$ \\
\hline Th-228 & $9.79 E-09$ & 1.75E-08 & 3.11E+08 & $5.45 E+\infty$ & $0 \%$ \\
\hline U-232 & 1.82E-07 & 3.26E-07 & $2.86 E+07$ & 9.32E +00 & $0 \%$ \\
\hline
\end{tabular}




\section{PUREX RADIONUCLIDE INGESTION DOSE CONTRIBUTION}

Mark 31B Fuat Assambly

5 years

Normalized

Isotope Isotopic Content Sludge Content Dose Conversion Dose Percent (g/assembly) (g/gPu-239) (ICRP-30, rem/g) (rem/g Pu-239) Dose

\begin{tabular}{|c|c|c|c|c|c|}
\hline U-234 & 9.71E-18 & $1.74 \mathrm{E}-17$ & $1.62 E+03$ & $2.81 E-14$ & $0 \%$ \\
\hline U-235 & 7.95E-02 & 1.42E-01 & 5.41E-01 & 7.œ1E-02 & $0 \%$ \\
\hline U-236 & 2.75E-03 & 4.93E-03 & $1.62 E+01$ & $7.98 E-02$ & $0 \%$ \\
\hline U-238 & 4.77E+01 & 8.53E+01 & 7.73E-02 & $6.60 E+00$ & $0 \%$ \\
\hline Np-237 & 7.47E-03 & 1.34E-02 & $2.75 E+03$ & 3.67E+01 & $0 \%$ \\
\hline Pu-236 & 9.38E-08 & $1.68 \mathrm{E}-07$ & $6.93 E+08$ & $1.16 E+02$ & $0 \%$ \\
\hline Pu-238 & 6.68E-05 & $1.20 E-04$ & $6.51 E+07$ & $7.78 E+03$ & $0 \%$ \\
\hline Pu-239 & 5.59E-01 & $1.00 E+00$ & 2.67E+05 & $2.67 E+05$ & $0 \%$ \\
\hline Pu-240 & 3.41E-02 & 6.11E-02 & $9.75 E+05$ & $5.95 E+04$ & $0 \%$ \\
\hline Pu-241 & $3.24 E-03$ & $5.79 E-03$ & $8.86 E+06$ & $5.13 E+04$ & $0 \%$ \\
\hline Pu-242 & $1.49 E-0.4$ & 2.67E-0.4 & $1.61 E+04$ & $4.30 \mathrm{E}+00$ & $0 \%$ \\
\hline Am-241 & 4.56E-02 & 8.16E-02 & $1.54 E+07$ & $1.26 E+06$ & $2 \%$ \\
\hline Am-242m & 2.02E-05 & 3.ळ2E-0.5 & $4.08 E+07$ & $1.48 E+03$ & $0 \%$ \\
\hline Am-243 & 2.29E-06 & 4.10E-06 & $8.97 E+05$ & $3.68 E+00$ & $0 \%$ \\
\hline $\mathrm{Cm}-242$ & $1.09 E-07$ & 1.94E-07 & $3.64 E+08$ & $7.06 E+01$ & $0 \%$ \\
\hline $\mathrm{Cm}-243$ & 2.20E-07 & 3.94E-07 & $1.50 E+08$ & $5.91 E+01$ & $0 \%$ \\
\hline $\mathrm{Cm}-244$ & $3.06 E-06$ & 5.47E-06 & $1.86 E+08$ & $1.02 E+03$ & $0 \%$ \\
\hline$C m-245$ & 1.37E-10 & 2.45E-10 & $7.73 E+05$ & $1.89 E-04$ & $0 \%$ \\
\hline $\mathrm{Cm}-246$ & 1.41E-12 & 2.53E-12 & $1.38 E+06$ & $3.49 E-06$ & $0 \%$ \\
\hline $\mathrm{Cm}-247$ & 1.14E-15 & $2.04 E-15$ & $3.80 E+02$ & $7.75 E-13$ & $0 \%$ \\
\hline $\mathrm{Cm}-248$ & $5.02 E-18$ & 8.98E-18 & $6.80 E+04$ & $6.11 E-13$ & $0 \%$ \\
\hline Bk-249 & 2.84E-24 & 5.07E-24 & $1.00 E+07$ & 5.07E-17 & $0 \%$ \\
\hline \multirow[t]{2}{*}{ Cf-249 } & 1.56E-22 & 2.78E-22 & $1.84 E+07$ & 5.12E-15 & $0 \%$ \\
\hline & & & & $6.63 E+07$ & \\
\hline
\end{tabular}


PUREX RADIONUCUDE INGESTION DOSE CONTRIBUTION

\begin{tabular}{|c|c|c|c|c|c|}
\hline Isotspe & $\begin{array}{l}\text { Isotopic Content } \\
\text { (g/assembly) }\end{array}$ & $\begin{array}{l}\text { Normalized } \\
\text { Sludge Content } \\
\text { ( } g / g \text { Pu-239) }\end{array}$ & $\begin{array}{l}\text { Dose Comversion } \\
\text { (lCRP-30, rem/g) }\end{array}$ & $\begin{array}{l}\text { Dose } \\
\text { rem/g Pu-239) }\end{array}$ & $\begin{array}{l}\text { Percent } \\
\text { Dose }\end{array}$ \\
\hline H3 & $1.76 \mathrm{E}-06$ & $0.00 \mathrm{E}+00$ & 6.11E+05 & $0.00 \mathrm{E}+00$ & $0 \%$ \\
\hline$C_{-}-51$ & $2.89 E-63$ & 4.91E-63 & $1.20 E+07$ & 5.89E-56 & $0 \%$ \\
\hline Co-60 & 4.08E-04 & 7.30E-04 & 2.94E+07 & 2.15E+04 & $0 \%$ \\
\hline Se-79 & $1.84 E-02$ & 2.96E-02 & $5.56 E+02$ & $1.64 E+01$ & $0 \%$ \\
\hline $\mathrm{Rb}-87$ & 7.83E-01 & 7.01E-02 & 4.17E-0.4 & 2.92E-05 & $0 \%$ \\
\hline$S r-89$ & 2.23E-32 & $4.00 \mathrm{E}-32$ & 2.53E+08 & 1.01E-23 & $0 \%$ \\
\hline Stree & $1.28 E+\infty 0$ & $2.28 E+00$ & $1.81 E_{+07}$ & $4.13 E+07$ & $87 \%$ \\
\hline$Y=90$ & $3.32 E-04$ & 5.94E-04 & $5.43 E+09$ & $3.23 E+06$ & $7 \%$ \\
\hline$Y-91$ & 1.11E-28 & $1.99 E-28$ & $2.18 E+08$ & 4.33E-20 & $0 \%$ \\
\hline$Z_{r-93}$ & $2.28 E+00$ & $4.07 E+00$ & $4.02 E+00$ & $1.64 E+01$ & $0 \%$ \\
\hline Zr-95 & 1.35E-25 & $2.42 E-25$ & $7.30 E+07$ & $1.77 \mathrm{E}-17$ & $0 \%$ \\
\hline $\mathrm{Nb}-95$ & 1.56E-25 & $2.80 E-25$ & $8.63 E+07$ & $2.41 E-17$ & $0 \%$ \\
\hline $\mathrm{Nb}-95 \mathrm{~m}$ & $9.47 E-29$ & $1.69 E-28$ & $7.35 E+08$ & 1.25E-19 & $0 \%$ \\
\hline Tc-99 & $2.65 E+00$ & $0.00 E+00$ & $2.20 E+01$ & $0.00 E+\infty$ & $0 \%$ \\
\hline$R u-103$ & $3.35 E-42$ & $3.00 E-42$ & $8.70 E+07$ & 2.61E-34 & $0 \%$ \\
\hline$R u-106$ & $2.51 E-05$ & $2.24 E-0.5$ & $7.03 E+07$ & $1.58 \mathrm{E}+03$ & $0 \%$ \\
\hline Pd-107 & 5.12E-01 & $9.16 E-01$ & 7.20E-02 & $6.60 E-02$ & $0 \%$ \\
\hline $\mathrm{Cd}-115 \mathrm{~m}$ & $3.10 E-40$ & $5.28 E-40$ & 3.81E+08 & 2.01E-31 & $0 \%$ \\
\hline $\ln -115$ & $2.60 E-02$ & 4.42E-02 & $7.28 \mathrm{E}-07$ & $3.21 E-08$ & $0 \%$ \\
\hline Sn-121m & $7.36 \mathrm{E}-06$ & $1.26 \mathrm{E}-0.5$ & $5.08 E+04$ & $6.42 E-01$ & $0 \%$ \\
\hline Sn-123 & $2.41 E-15$ & 4.15E-15 & $6.33 E+07$ & 2.63E-07 & $0 \%$ \\
\hline Sn-126 & 7.99E-02 & 1.37E-01 & $4.82 E+02$ & $6.62 E+01$ & $0 \%$ \\
\hline Sb-124 & $1.94 E-32$ & $3.45 E-32$ & $1.63 E+08$ & 5.63E-24 & $0 \%$ \\
\hline Sb-125 & $1.22 \mathrm{E}-03$ & $2.18 E-03$ & $2.69 E+06$ & $5.85 E+03$ & $0 \%$ \\
\hline Sb-126 & $3.80 E-10$ & 6.53E-10 & $6.10 E+06$ & $3.98 E-03$ & $0 \%$ \\
\hline Te-127m & 1.83E-17 & $3.28 E-17$ & $7.45 E+07$ & 2.44E-09 & $0 \%$ \\
\hline To-129m & 4.16E-51 & $7.44 E-51$ & $2.98 E+08$ & 2.22E-42 & $0 \%$ \\
\hline $\mid-129$ & $5.60 E-01$ & $0.00 E+00$ & $4.94 E_{+01}$ & $0.00 E_{+}+\infty$ & $0 \%$ \\
\hline Cs-134 & $1.73 E-04$ & $1.55 E-05$ & $9.58 E+07$ & $1.49 E+03$ & $0 \%$ \\
\hline Cs-135 & 3.19E-01 & $2.86 \mathrm{E}-02$ & 8.17E+00 & 2.34E-01 & $0 \%$ \\
\hline Cs-137 & $2.70 E+00$ & 2.42E-01 & $4.33 E+06$ & $1.05 E+06$ & $2 \%$ \\
\hline$\overline{C o-141}$ & $4.82 E-51$ & $8.62 E-51$ & 7.41E+07 & $6.39 E-43$ & $0 \%$ \\
\hline Co-144 & $4.73 E-06$ & $8.46 E-06$ & $6.38 E+07$ & $5.40 E+02$ & $0 \%$ \\
\hline Nd-144 & $3.20 E+\infty 0$ & $5.73 E+\infty 0$ & $0.00 E+\infty 0$ & $0.00 E+\infty 0$ & $0 \%$ \\
\hline Pm-147 & $2.58 E-02$ & 4.ळE-02 & $8.81 E+05$ & $4.07 E+04$ & $0 \%$ \\
\hline$P m-148$ & $8.72 E-45$ & $1.56 \mathrm{E}-44$ & $1.56 E+09$ & 2.43E-35 & $0 \%$ \\
\hline Pm-148m & $9.72 E-43$ & $1.74 E-42$ & $1.50 E+08$ & 2.61E-34 & $0 \%$ \\
\hline Sm-147 & $1.34 E+\infty$ & $2.39 E+\infty 0$ & $4.19 E-03$ & $1.00 \mathrm{E}-02$ & $0 \%$ \\
\hline Sm-151 & $1.63 E-01$ & $2.92 E-01$ & $8.95 E+03$ & $2.61 E_{+} 03$ & $0 \%$ \\
\hline Eu-152 & 2.21E-04 & $3.96 E-0.4$ & $1.04 E+06$ & $4.12 E+02$ & $0 \%$ \\
\hline Eu-154 & $3.05 E-03$ & $5.46 E-03$ & 2.40E+06 & $1.31 E+04$ & $0 \%$ \\
\hline Eu-155 & $4.40 \mathrm{E}-03$ & 7.87E-03 & $6.05 E+05$ & $4.76 E+03$ & $0 \%$ \\
\hline Gd-152 & $1.06 \mathrm{E}-05$ & $1.89 E-05$ & $3.22 E-06$ & $6.08 E-11$ & $0 \%$ \\
\hline Tb-160 & $7.52 E-28$ & 1.34E-27 & $7.23 E+07$ & $9.72 E-20$ & $0 \%$ \\
\hline $\mathrm{Ra}-224$ & $3.29 E-11$ & $5.89 E-11$ & $5.31 E+10$ & $3.13 E+00$ & $0 \%$ \\
\hline Th-228 & $6.40 \mathrm{E}-09$ & $1.14 E-08$ & $3.11 E+08$ & $3.56 E+00$ & $0 \%$ \\
\hline U-232 & 2.44E-07 & 4.37E-07 & $2.86 E+07$ & $1.25 E+01$ & $0 \%$ \\
\hline$U-234$ & 9.70 E-18 & $1.74 E-17$ & $1 . \ltimes 2 E+03$ & $2.81 E-14$ & $0 \%$ \\
\hline $4-235$ & 7.95E-02 & 1.42E-01 & 5.41E-01 & 7.69E-02 & $0 \%$ \\
\hline$U-236$ & $2.75 E-03$ & 4.93E-03 & $1.62 E+01$ & $7.98 E-02$ & $0 \%$ \\
\hline U-238 & 4.77E+01 & $8.54 E+01$ & 7.73E-02 & $6.60 \mathrm{E}+00$ & $0 \%$ \\
\hline Np-237 & 7.47E-03 & $1.34 E-02$ & $2.75 E+03$ & 3.67E+01 & $0 \%$ \\
\hline Pu-236 & 8.25E-09 & $1.48 E-08$ & $6.93 E+08$ & $1.02 E+01$ & $0 \%$ \\
\hline Pu-238 & 6.25E-05 & $1.12 E-04$ & $6.51 E+07$ & $7.28 E+03$ & $0 \%$ \\
\hline $\mathrm{Pu}=232$ & $5.59 E-01$ & $1.00 E+\infty 0$ & $2.67 E+05$ & 2.67E+05 & 18 \\
\hline
\end{tabular}




\section{PUREX RADIONUCLIDE INGESTION DOSE CONTRIBUTION}

Mark 31 B Fuol Assombly

15 yoars

Normalized

Isotope Isotopic Content Sludge Content Dose Corversion Dose Percent

(g/assembly) (g/g Pu-239) (ICRP-30, rem/g) (rem/g Pu-239) Dose

\begin{tabular}{llllll}
\hline Pu-240 & $3.41 E-02$ & $6.10 E-02$ & $9.75 E+05$ & $5.95 E+04$ & $0 \%$ \\
Pu-241 & $2.02 E-03$ & $3.6 E-03$ & $8.86 E+06$ & $3.20 E+04$ & $0 \%$ \\
Pu-242 & $1.49 E-04$ & $2.67 E-04$ & $1.61 E+04$ & $4.30 E+00$ & $0 \%$ \\
Am-241 & $4.61 E-02$ & $8.25 E-02$ & $1.54 E+07$ & $1.27 E+06$ & $3 \%$ \\
Am-242m & $1.93 E-05$ & $3.46 E-05$ & $4.08 E+07$ & $1.41 E+03$ & $0 \%$ \\
Am-243 & $2.29 E-06$ & $4.10 E-06$ & $8.97 E+05$ & $3.68 E+00$ & $0 \%$ \\
Cm-242 & $4.68 E-08$ & $8.38 E-08$ & $3.64 E+08$ & $3.05 E+01$ & $0 \%$ \\
Cm-243 & $1.73 E-07$ & $3.09 E-07$ & $1.50 E+08$ & $4.64 E+01$ & $0 \%$ \\
Cm-244 & $2.09 E-08$ & $3.73 E-08$ & $1.86 E+08$ & $6.94 E+00$ & $0 \%$ \\
Cm-245 & $1.37 E-10$ & $2.45 E-10$ & $7.73 E+05$ & $1.89 E-04$ & $0 \%$ \\
Cm-246 & $1.41 E-12$ & $2.53 E-12$ & $1.38 E+06$ & $3.49 E-06$ & $0 \%$ \\
Cm-247 & $1.14 E-15$ & $2.04 E-15$ & $3.80 E+02$ & $7.75 E-13$ & $0 \%$ \\
Cm-248 & $5.02 E-18$ & $8.99 E-18$ & $6.80 E+04$ & $6.11 E-13$ & $0 \%$ \\
Bk-249 & $8.93 E-28$ & $1.60 E-27$ & $1.00 E+07$ & $1.60 E-20$ & $0 \%$ \\
Cf-249 & $1.55 E-22$ & $2.78 E-22$ & $1.84 E+07$ & $5.12 E-15$ & $0 \%$
\end{tabular}


Mark 31A

\begin{tabular}{|c|c|c|c|c|c|c|c|c|c|c|c|c|c|c|}
\hline \multirow[b]{4}{*}{ Isotopo } & \multirow{2}{*}{\multicolumn{4}{|c|}{ INHALATION }} & \multirow{2}{*}{\multicolumn{2}{|c|}{ INGESTION }} & \multirow{4}{*}{$\begin{array}{c}\text { Volatile } \\
\text { Inh/lng } \\
\text { ratio }\end{array}$} & \multirow{2}{*}{\multicolumn{4}{|c|}{ INHALATION }} & \multirow{2}{*}{\multicolumn{2}{|c|}{ INGESTION }} & \multirow{4}{*}{$\begin{array}{c}\text { Volatile } \\
\text { Inh/lng } \\
\text { ratio }\end{array}$} \\
\hline & & & & & & & & & & & & & & \\
\hline & \multicolumn{2}{|c|}{ Volatile Conditions } & \multicolumn{2}{|c|}{ Evaporativo Conditions } & \multicolumn{2}{|c|}{ Volatile Conditions } & & Volatile Con & ditions & Evaporative & Sonditions & Volatile Co & ditions & \\
\hline & $\begin{array}{c}\text { Doso } \\
\text { (rem/g Pu-239) }\end{array}$ & $\begin{array}{l}\text { Percent } \\
\text { Dose }\end{array}$ & $\begin{array}{c}\text { Dose } \\
(\operatorname{rom} / 9 \mathrm{Pu}-239)\end{array}$ & $\begin{array}{c}\text { Percant } \\
\text { Dose }\end{array}$ & $\begin{array}{c}\text { Dose } \\
\text { (rem/g Pu-239) }\end{array}$ & $\begin{array}{l}\text { Percent } \\
\text { Dose }\end{array}$ & & $\begin{array}{c}\text { Dose } \\
(r \in m / g \text { Pu-239) }\end{array}$ & $\begin{array}{l}\text { Percent } \\
\text { Dose }\end{array}$ & $\begin{array}{c}\text { Dose } \\
\text { (rem/g Pu-239) }\end{array}$ & $\begin{array}{c}\text { Percent } \\
\text { Dose }\end{array}$ & $\begin{array}{c}\text { Dose } \\
\text { (rem/g Pu-239) }\end{array}$ & $\begin{array}{l}\text { Percent } \\
\text { Dose }\end{array}$ & \\
\hline H-3 & $0.00 E+\infty$ & $0 \%$ & $0.00 E+\infty$ & $0 \%$ & $0.00 E+00$ & $0 \%$ & - & $0.00 E+00$ & $0 \%$ & $0.00 E+\infty$ & $0 \%$ & $0.00 \mathrm{E}+\infty$ & $0 \%$ & - \\
\hline Cr-51 & 8.66E-16 & $0 \%$ & 8.66E-19 & $0 \%$ & 4.33E-16 & $0 \%$ & 2 & 2.44E-55 & $0 \%$ & 2.44E-58 & $0 \%$ & 1.22E-55 & $0 \%$ & 2 \\
\hline Co-60 & $9.61 E+05$ & $0 \%$ & $9.61 E+02$ & $0 \%$ & $1.66 E+05$ & $0 \%$ & 6 & $2.57 E+05$ & $0 \%$ & $2.57 \mathrm{E}+02$ & $0 \%$ & $4.45 E+04$ & $0 \%$ & 6 \\
\hline Se-79 & $1.76 E+01$ & $0 \%$ & $1.76 E-01$ & $0 \%$ & $1.58 E+01$ & $0 \%$ & 1 & $1.76 E+01$ & $0 \%$ & 1.76E-01 & $0 \%$ & $1.58 E+01$ & $0 \%$ & 1 \\
\hline$R b-87$ & $1.88 E-04$ & $0 \%$ & $1.88 E-04$ & $0 \%$ & 2.73E-05 & $0 \%$ & 7 & 3.76E-04 & $0 \%$ & 3.76E-07 & $0 \%$ & $2.73 E-05$ & $0 \%$ & 14 \\
\hline Sr-89 & $5.20 E-02$ & $0 \%$ & $5.20 E-05$ & $0 \%$ & $1.23 \mathrm{E}-02$ & $0 \%$ & 4 & $3.73 E-23$ & $0 \%$ & 3.73E-26 & $0 \%$ & 8.82E-24 & $0 \%$ & 4 \\
\hline Sr.90 & $4.92 E+08$ & $60 \%$ & $4.92 E+05$ & 448 & $4.92 E+07$ & 79\% & 10 & $3.85 E+08$ & $64 \%$ & $3.85 E+05$ & $63 \%$ & $3.85 E+07$ & $87 \%$ & 10 \\
\hline Y-90 & $3.15 E+06$ & $0 \%$ & $3.15 E+03$ & $0 \%$ & $3.84 E+06$ & $6 \%$ & 1 & $2.47 E+06$ & $0 \%$ & $2.47 E+03$ & $0 \%$ & $3.00 E+06$ & $7 \%$ & 1 \\
\hline Y-91 & $1.09 E+\infty 0$ & $0 \%$ & $1.09 E-03$ & $0 \%$ & 2.21E-01 & $0 \%$ & 5 & 1.89E-19 & $0 \%$ & $1.89 E-22$ & $0 \%$ & 3.81E-20 & $0 \%$ & 5 \\
\hline $2 r-83$ & $3.08 E+03$ & $0 \%$ & $3.08 E+\infty$ & $0 \%$ & $1.54 E+01$ & $0 \%$ & 200 & $3.0 \pi E+03$ & $0 \%$ & $3.07 E+00$ & $0 \%$ & $1.54 E+01$ & $0 \%$ & 200 \\
\hline Zr-95 & $5.12 E+\infty$ & $0 \%$ & $5.12 E-03$ & $0 \%$ & 8.68E-01 & $0 \%$ & 5 & 8.37E-17 & $0 \%$ & 8.37E-20 & $0 \%$ & 1.58E-17 & $0 \%$ & 5 \\
\hline $\mathrm{Nb}-95$ & $2.70 E+00$ & $0 \%$ & $2.70 E-03$ & $0 \%$ & $1.32 E+\infty$ & $0 \%$ & 2 & $4.41 \mathrm{E}-17$ & $0 \%$ & 4.41E-20 & $0 \%$ & 2.16E-17 & $0 \%$ & 2 \\
\hline $\mathrm{Nb}-95 \mathrm{~m}$ & $7.50 E-03$ & $0 \%$ & 7.50 E- 06 & $0 \%$ & $6.82 E-03$ & $0 \%$ & 1 & 1.23E-19 & $0 \%$ & $1.23 \mathrm{E}-22$ & $0 \%$ & 1.12E-19 & $0 \%$ & 1 \\
\hline TC-98 & $0.00 E+\infty$ & $0 \%$ & $0.00 E+00$ & $0 \%$ & $0.00 E+\infty 0$ & $0 \%$ & - & $0.00 \mathrm{E}+\infty$ & $0 \%$ & $0.00 E+\infty 0$ & $0 \%$ & $0.00 E+\infty 0$ & $0 \%$ & - \\
\hline$R u-103$ & 3.84E-06 & $0 \%$ & $3.94 E-08$ & $0 \%$ & $1.37 E-06$ & $0 \%$ & 3 & $6.76 E-34$ & $0 \%$ & $6.76 \mathrm{E}-36$ & $0 \%$ & 2.34E-34 & $0 \%$ & 3 \\
\hline$R u-106$ & $3.12 E+07$ & 4\% & $3.12 E+05$ & $28 \%$ & $1.49 E+06$ & 28 & 21 & $3.2 \pi E+04$ & $0 \%$ & $3.27 E+02$ & $0 \%$ & $1.57 E+03$ & $0 \%$ & 21 \\
\hline Pd-107 & $6.10 E+\infty 0$ & $0 \%$ & $6.10 E-03$ & $0 \%$ & 6.5TE-02 & $0 \%$ & 93 & $6.10 \mathrm{E}+\infty 0$ & $0 \%$ & $6.10 E-03$ & $0 \%$ & $6.58 E-02$ & $0 \%$ & 93 \\
\hline Cd-115m & 4.21E-06 & $0 \%$ & $4.21 E-09$ & $0 \%$ & 8.73E-07 & $0 \%$ & 4 & 9.37E-31 & $0 \%$ & 9.37E-34 & $0 \%$ & 2.16E-31 & $0 \%$ & 4 \\
\hline $\ln -115$ & 8.96E-07 & $0 \%$ & 8.86E-10 & $0 \%$ & 3.69E-08 & $0 \%$ & 24 & 8.87E- -07 & $0 \%$ & 8.97E-10 & $0 \%$ & $3.66 E-08$ & $0 \%$ & 25 \\
\hline $\mathrm{Sn}-121 \mathrm{~m}$ & $4.91 E+\infty 0$ & $0 \%$ & 4.91E-03 & $0 \%$ & 7.17E-01 & $0 \%$ & 7 & $4.28 E+\infty 0$ & $0 \%$ & $4.28 E-03$ & $0 \%$ & $6.24 E-01$ & $0 \%$ & 7 \\
\hline Sn-123 & $3.74 E+02$ & $0 \%$ & $3.74 E-01$ & $0 \%$ & $8.5 \pi+01$ & $0 \%$ & 4 & 1.12E-06 & $0 \%$ & 1.12E-09 & $0 \%$ & 2.87E-07 & $0 \%$ & 4 \\
\hline$S n-125$ & 2.01E-50 & $0 \%$ & 2.01E-53 & $0 \%$ & $1.51 E-52$ & $0 \%$ & 133 & $0.00 E+00$ & $0 \%$ & $0.00 E+00$ & $0 \%$ & $0.00 E+\infty$ & $0 \%$ & - \\
\hline Sn-126 & $3.53 E+02$ & $0 \%$ & 3.53E-01 & $0 \%$ & $6.87 E+01$ & $0 \%$ & 5 & $3.53 E+02$ & $0 \%$ & 3.53E-01 & $0 \%$ & $6.97 E+01$ & $0 \%$ & 5 \\
\hline Sb-124 & 2.78E-05 & $0 \%$ & $2.70 E-08$ & $0 \%$ & 1.24E-05 & $0 \%$ & 2 & $1.52 E-23$ & $0 \%$ & $1.52 E-26$ & $0 \%$ & 6.74E-24 & $0 \%$ & 2 \\
\hline Sb-125 & $3.01 E+05$ & $0 \%$ & $3.01 E+02$ & $0 \%$ & $8.03 E+04$ & $0 \%$ & 4 & $2.38 E+04$ & $0 \%$ & $2.38 E+01$ & $0 \%$ & $6.34 E+03$ & $0 \%$ & 4 \\
\hline Sb-126 & $5.75 E-01$ & $0 \%$ & 5.75E-04 & $0 \%$ & $4.19 \mathrm{E}-03$ & $0 \%$ & 137 & $5.75 E-01$ & $0 \%$ & $5.75 E-04$ & $0 \%$ & 4.19E-03 & $0 \%$ & 137 \\
\hline To-127m & $7.09 E+01$ & $0 \%$ & 7.09E-01 & $0 \%$ & $2.85 E+01$ & $0 \%$ & 2 & 5.80E-09 & $0 \%$ & $5.80 \mathrm{E}-11$ & $0 \%$ & 2.41E-09 & $0 \%$ & 2 \\
\hline To-120m & 3.34E-09 & $0 \%$ & 3.34E-11 & $0 \%$ & $1.65 E-09$ & $0 \%$ & 2 & $4.02 E-42$ & $0 \%$ & $4.02 E-44$ & $0 \%$ & $1.89 E-42$ & $0 \%$ & 2 \\
\hline$-12 \theta$ & $0.00 E+00$ & $0 \%$ & $1.57 E+01$ & $0 \%$ & $0.00 E+\infty 0$ & $0 \%$ & - & $0.00 E+\infty 0$ & $0 \%$ & $0.00 E+\infty 0$ & $0 \%$ & $0.00 E+\infty 0$ & $0 \%$ & - \\
\hline |-131 & $0.00 E+\infty 0$ & $0 \%$ & $2.97 E+02$ & $0 \%$ & $0.00 E+00$ & $0 \%$ & - & $0.00 E+\infty 0$ & $0 \%$ & $0.00 E+00$ & $0 \%$ & $0.00 E+00$ & $0 \%$ & - \\
\hline C8-134 & 2.44E+04 & $0 \%$ & $2.44 E+02$ & $0 \%$ & $3.84 E+04$ & $0 \%$ & 1 & $1.11 E_{+} 03$ & $0 \%$ & $1.11 E+01$ & $0 \%$ & $1.74 E+03$ & $0 \%$ & 1 \\
\hline C8-135 & $1.92 E-01$ & $0 \%$ & $1.92 E-03$ & $0 \%$ & 3.03E-01 & $0 \%$ & 1 & $1.82 E-01$ & $0 \%$ & $1.92 \mathrm{E}-03$ & $0 \%$ & 3.03E-01 & $0 \%$ & 1 \\
\hline C8-136 & 2.35E-37 & $0 \%$ & 2.35E-39 & $0 \%$ & 3.45E-37 & $0 \%$ & 1 & $0.00 E+\infty 0$ & $0 \%$ & $0.00 E+00$ & $0 \%$ & $0.00 E+00$ & $0 \%$ & - \\
\hline C. -137 & $7.99 E+05$ & $0 \%$ & $7.99 E+03$ & 18 & $1.25 E+06$ & 28 & 1 & $6.35 E+05$ & $0 \%$ & $6.35 E+03$ & 18 & $9.82 E+05$ & $2 \%$ & 1 \\
\hline$B a-140$ & $6.14 E-35$ & $0 \%$ & $6.14 E-38$ & $0 \%$ & $1.43 E-34$ & $\overline{0 \%}$ & 0 & $0.00 E+00$ & $0 \%$ & $0.00 E+\infty 0$ & $0 \%$ & $0.00 E+\infty$ & $0 \%$ & - \\
\hline La-140 & 8.62E-35 & $0 \%$ & 8.63E-38 & $0 \%$ & $1.51 E-34$ & $0 \%$ & 1 & $0.00 E+\infty 0$ & $0 \%$ & $0.00 E+00$ & $0 \%$ & $0.00 E+\infty 0$ & $0 \%$ & - \\
\hline Co-141 & 1.13E-08 & $0 \%$ & 1.13E-11 & $0 \%$ & $3.46 \mathrm{E}-09$ & $0 \%$ & 3 & 1.79E-42 & $0 \%$ & $1.79 E-45$ & $0 \%$ & $5.49 E-43$ & $0 \%$ & 3 \\
\hline Co-144 & $6.47 E+07$ & B\% & $6.47 E+04$ & $6 x$ & $3.69 E+06$ & $6 x$ & 18 & $8.81 E+\infty 3$ & $0 \%$ & $8.81 E+\infty$ & $0 \%$ & $5.02 E+02$ & $0 \%$ & 18 \\
\hline$P\{-143$ & $3.82 \mathrm{E}-32$ & $0 \%$ & $3.82 E-35$ & $0 \%$ & $2.35 E-32$ & $0 \%$ & 2 & $0.00 E+\infty$ & $0 \%$ & $0.00 E+\infty$ & $0 \%$ & $0.00 E+\infty 0$ & $0 \%$ & - \\
\hline $\mathrm{Nd}-144$ & $0.00 E+00$ & $0 \%$ & $0.00 E+\infty 0$ & $0 \%$ & $0.00 \mathrm{E}+00$ & $0 \%$ & - & $0.00 E+\infty 0$ & $0 \%$ & $0.00 E+\infty 0$ & $0 \%$ & $0.00 E+00$ & $0 \%$ & - \\
\hline Nd-147 & $3.72 E-42$ & $0 \%$ & 3.72E-45 & $0 \%$ & $2.34 \mathrm{E}-42$ & $0 \%$ & 2 & $0.00 E+\infty 0$ & $0 \%$ & $0.00 E+\infty 0$ & $0 \%$ & $0.00 E+00$ & $0 \%$ & - \\
\hline$P m-147$ & $1.91 E+07$ & $2 \%$ & $1.91 E+04$ & 22 & $5.33 E+05$ & $1 \%$ & 36 & $1.36 E+06$ & $0 \%$ & $1.36 E+03$ & $0 \%$ & $3.78 E+04$ & $0 \%$ & 36 \\
\hline
\end{tabular}


RADIONUCLIDE DOSE CONTAIBUTION COMPARISON

Mark $31 A$

\begin{tabular}{|c|c|c|c|c|c|c|c|c|c|c|c|c|c|c|}
\hline \multirow[b]{4}{*}{ Isotope } & \multirow{2}{*}{\multicolumn{4}{|c|}{ INHALATION }} & \multirow{2}{*}{\multicolumn{2}{|c|}{ INGESTION }} & \multirow{4}{*}{$\begin{array}{c}\text { Volatilo } \\
\text { Inh/ling } \\
\text { ratio }\end{array}$} & \multirow{2}{*}{\multicolumn{4}{|c|}{ INHALATION }} & \multirow{2}{*}{\multicolumn{2}{|c|}{ INGESTION }} & \multirow{4}{*}{$\begin{array}{c}\text { Volatije } \\
\text { Inh/hing } \\
\text { ratio }\end{array}$} \\
\hline & & & & & & & & & & & & & & \\
\hline & \multicolumn{2}{|c|}{ Volatilo Conditions } & \multicolumn{2}{|c|}{ Evaporative Conditions } & \multicolumn{2}{|c|}{ Volatile Conditions } & & Volatile Co & ditions & Eveporative & Conditions & Volatile Co & ditions & \\
\hline & $\begin{array}{c}\text { Dose } \\
(r \mathrm{rem} / \mathrm{g} \text { Pu-239) }\end{array}$ & $\begin{array}{l}\text { Percont } \\
\text { Dose }\end{array}$ & $\begin{array}{c}\text { Dose } \\
(r e m / 9 \text { Pu-239) }\end{array}$ & $\begin{array}{c}\text { Percent } \\
\text { Dose }\end{array}$ & $\begin{array}{c}\text { Dose } \\
(\text { rem/g Pu-239) }\end{array}$ & $\begin{array}{l}\text { Percent } \\
\text { Dose }\end{array}$ & & $\begin{array}{c}\text { Dose } \\
(r \in m / g \text { Pu-239) }\end{array}$ & $\begin{array}{c}\text { Percent } \\
\text { Dose }\end{array}$ & $\begin{array}{c}\text { Dose } \\
\text { (rem/g Pu-239) }\end{array}$ & $\begin{array}{c}\text { Percent } \\
\text { Dose }\end{array}$ & $\begin{array}{c}\text { Dose } \\
(r e m / g \text { Pu-239) }\end{array}$ & $\begin{array}{c}\text { Percent } \\
\text { Dose }\end{array}$ & \\
\hline$P m-148$ & $1.37 \mathrm{E}-08$ & $0 \%$ & $1.37 \mathrm{E}-11$ & $0 \%$ & $1.30 \mathrm{E}-0 \mathrm{~B}$ & $0 \%$ & 1 & $3.27 \mathrm{E}-35$ & $0 \%$ & $3.27 \mathrm{E}-38$ & $0 \%$ & $3.11 \mathrm{E}-35$ & $0 \%$ & 1 \\
\hline$P m-148 m$ & $1.99 €-07$ & $0 \%$ & $1.99 \mathrm{E}-10$ & $0 \%$ & $1.40 E-07$ & $0 \%$ & 1 & $4.75 E-34$ & $0 \%$ & $4.75 E-37$ & $0 \%$ & $3.33 E-34$ & $0 \%$ & 1 \\
\hline$S m-147$ & $2.76 E+\infty 0$ & $0 \%$ & $2.76 E-03$ & $0 \%$ & $7.02 E-03$ & $0 \%$ & 394 & $3.69 E+\infty 0$ & $0 \%$ & $3.69 E-03$ & $0 \%$ & 9.37E-03 & $0 \%$ & 394 \\
\hline Sm-151 & $2.48 E+05$ & $0 \%$ & $2.48 E+02$ & $0 \%$ & $2.91 E+03$ & $0 \%$ & 85 & $2.30 E+05$ & $0 \%$ & $2.30 E+02$ & $0 \%$ & $2.70 E+03$ & $0 \%$ & 85 \\
\hline Eu-152 & $2.32 E+04$ & $0 \%$ & $2.32 E+01$ & $0 \%$ & $6.33 E+02$ & $0 \%$ & 37 & $1.36 E+04$ & $0 \%$ & $1.36 E+01$ & $0 \%$ & $3.71 E+02$ & $0 \%$ & 37 \\
\hline$E u-154$ & $8.89 E+05$ & $0 \%$ & $8.89 E+02$ & $0 \%$ & $3.11 E+04$ & $0 \%$ & 29 & $3.97 E+05$ & $0 \%$ & $3.97 \mathrm{E}+02$ & $0 \%$ & $1.39 E+04$ & $0 \%$ & 29 \\
\hline Eu-155 & $6.26 E+05$ & $0 \%$ & $6.26 \mathrm{E}+02$ & $0 \%$ & $2.09 E+04$ & $0 \%$ & 30 & $1.48 E+05$ & $0 \%$ & $1.48 E+02$ & $0 \%$ & $4.94 E+03$ & $0 \%$ & 30 \\
\hline Eu-156 & $3.04 \mathrm{E}-29$ & $0 \%$ & $3.05 E-32$ & $0 \%$ & 2.41E-29 & $0 \%$ & 1 & $0.00 E+\infty$ & $0 \%$ & $0.00 E+\infty$ & $0 \%$ & $0.00 E+\infty$ & $0 \%$ & - \\
\hline$G \alpha-152$ & 1.13E-07 & $0 \%$ & 1.13E-10 & $0 \%$ & $7.08 E-11$ & $0 \%$ & 1599 & $1.13 E-07$ & $0 \%$ & 1.13E-10 & $0 \%$ & $7.08 E-11$ & $0 \%$ & 1599 \\
\hline Tb-160 & $6.61 E-04$ & $0 \%$ & 6.61E-07 & $0 \%$ & $1.93 E-04$ & $0 \%$ & 3 & $4.10 E-19$ & $0 \%$ & 4.10E-22 & $0 \%$ & 1.19E-19 & $0 \%$ & 3 \\
\hline Ra-224 & 2.53E-03 & $0 \%$ & 2.53E-06 & $0 \%$ & $2.88 E-04$ & $0 \%$ & 9 & $1.65 E-03$ & $0 \%$ & 1.65E-06 & $0 \%$ & $1.87 \mathrm{E}-04$ & $0 \%$ & 8 \\
\hline Th-228 & $2.67 \mathrm{E}-01$ & $0 \%$ & 2.67E-04 & $0 \%$ & $3.27 E-04$ & $0 \%$ & 817 & $1.74 E-01$ & $0 \%$ & $1.74 E-04$ & $0 \%$ & 2.13E-04 & $0 \%$ & 817 \\
\hline U-232 & 2.89E-01 & $0 \%$ & 2.89E-04 & $0 \%$ & $5.59 \mathrm{E}-04$ & $0 \%$ & 517 & $3.87 E-01$ & $0 \%$ & $3.87 E-04$ & $0 \%$ & $7.49 \mathrm{E}-04$ & $0 \%$ & 517 \\
\hline U.234 & $1.45 E-11$ & $0 \%$ & $1.45 E-14$ & $0 \%$ & $2.80 E-14$ & $0 \%$ & 499 & $1.45 E-11$ & $0 \%$ & $1.45 E-14$ & $0 \%$ & $2.90 \mathrm{E}-14$ & $0 \%$ & 499 \\
\hline U.235 & $3.90 E+01$ & $0 \%$ & 3.90E-02 & $0 \%$ & $8.15 E-02$ & $0 \%$ & 479 & $3.90 E+01$ & $0 \%$ & $3.80 E+01$ & $0 \%$ & 8.16E-02 & $0 \%$ & 478 \\
\hline U-236 & $3.64 E+01$ & $0 \%$ & $3.64 E-02$ & $0 \%$ & $7.60 E-02$ & $0 \%$ & 479 & $3.64 E+01$ & $0 \%$ & $3.64 E-02$ & $0 \%$ & $7.61 E-02$ & $0 \%$ & 478 \\
\hline U-238 & $3.56 E+03$ & $0 \%$ & $3.56 E+\infty$ & $0 \%$ & $6.82 E+\infty 0$ & $0 \%$ & 521 & $3.56 E+03$ & $0 \%$ & $3.56 E+\infty$ & $0 \%$ & $6.82 E+\infty$ & $0 \%$ & 521 \\
\hline Np-237 & $5.25 E+\infty 3$ & $0 \%$ & $5.25 E+\infty$ & $0 \%$ & 4.18E+01 & $0 \%$ & 125 & $5.25 E+03$ & $0 \%$ & $5.25 E+\infty$ & $0 \%$ & $4.19 E+01$ & $0 \%$ & 125 \\
\hline Pu-236 & 8.58E-01 & $0 \%$ & 8.59E-04 & $0 \%$ & $6.97 E-03$ & $0 \%$ & 123 & $7.55 E-02$ & $0 \%$ & 7.55E-05 & $0 \%$ & $6.13 E-04$ & $0 \%$ & 123 \\
\hline Pu-238 & $1.25 E+06$ & $0 \%$ & $1.25 E+03$ & $0 \%$ & $1.03 E+04$ & $0 \%$ & 121 & $1.17 E+06$ & $0 \%$ & $1.17 E+03$ & $0 \%$ & $9.65 E+03$ & $0 \%$ & 121 \\
\hline Pu-239" & $3.16 E+07$ & $4 \%$ & $3.16 \mathrm{E}+04$ & $3 x$ & $2.67 E+05$ & $0 \%$ & 118 & $3.16 E+07$ & 5\% & $3.16 E+04$ & $5 \%$ & $2.67 E+05$ & $1 \%$ & 118 \\
\hline Pu-240 & $6.68 E+06$ & $1 x$ & $6.68 E+03$ & $0 \%$ & $5.61 E+04$ & $0 \%$ & 119 & $6.67 E+06$ & $1 \%$ & $6.67 E+03$ & $\mathbf{1 x}$ & $5.61 E+04$ & $0 \%$ & 119 \\
\hline Pu-241 & $6.62 E+06$ & $1 \%$ & $6.62 E+03$ & $1 x$ & $5.70 E+04$ & $0 \%$ & 116 & $4.13 E+06$ & 1\% & $4.13 E+03$ & $1 x$ & $3.56 E+04$ & $0 \%$ & 116 \\
\hline Pu-242 & $5.06 E+02$ & $0 \%$ & 5.07E-01 & $0 \%$ & $4.31 E+\infty 0$ & $0 \%$ & 117 & $5.07 E+02$ & $0 \%$ & $5.07 \mathrm{E}-01$ & $0 \%$ & $4.32 E+\infty$ & $0 \%$ & 117 \\
\hline Am-241 & $1.65 E+08$ & $20 x$ & $1.65 E+05$ & $15 \%$ & $1.42 E+06$ & 28 & 116 & $1.67 E+08$ & $28 \%$ & $1.67 E+05$ & $27 x$ & $1.43 E+06$ & $3 x$ & 116 \\
\hline Amt-242m & $2.46 E+05$ & $0 \%$ & $2.49 E+02$ & $0 \%$ & $2.05 E+03$ & $0 \%$ & 122 & $2.38 E+05$ & $0 \%$ & $2.38 E+02$ & $0 \%$ & $1.96 E+03$ & $0 \%$ & 122 \\
\hline Am-243 & $5.29 E+02$ & $0 \%$ & 5.28E-01 & $0 \%$ & $4.56 E+00$ & $0 \%$ & 116 & $5.28 E+02$ & $0 \%$ & $5.28 E-01$ & $0 \%$ & $4.56 \mathrm{E}+\infty$ & $0 \%$ & 116 \\
\hline$C_{m-242}$ & $1.48 E+04$ & $0 \%$ & $1.48 E+01$ & $0 \%$ & $8.56 E+01$ & $0 \%$ & 155 & $6.54 E+03$ & $0 \%$ & $6.54 E+\infty$ & $0 \%$ & 4.23E+01 & $0 \%$ & 155 \\
\hline $\mathrm{Cm}_{\mathrm{m}}-243$ & $1.11 E+04$ & $0 \%$ & $1.11 E+01$ & $0 \%$ & $9.21 E+01$ & $0 \%$ & 121 & 8.72E +03 & $0 \%$ & 8.72E $+\infty$ & $0 \%$ & $7.23 E+01$ & $0 \%$ & 121 \\
\hline $\mathrm{Cm}-244$ & $1.78 E+03$ & $0 \%$ & $1.79 E+\infty 0$ & $0 \%$ & $1.52 E+01$ & $0 \%$ & 118 & $1.22 E+03$ & $0 \%$ & $1.22 E+\infty$ & $0 \%$ & $1.04 E+01$ & $0 \%$ & 117 \\
\hline $\mathrm{Cm}-245$ & 4.33E-02 & $0 \%$ & 4.33E-05 & $0 \%$ & $3.61 E-04$ & $0 \%$ & 120 & 4.33E-02 & $0 \%$ & 4.33E-05 & $0 \%$ & $3.61 E-04$ & $0 \%$ & 120 \\
\hline $\mathrm{Cm}-246$ & $7.28 E-04$ & $0 \%$ & 7.28E-07 & $0 \%$ & $6.09 E-06$ & $0 \%$ & 120 & 7.27E-04 & $0 \%$ & 7.27E-07 & $0 \%$ & $6.08 E-06$ & $0 \%$ & 120 \\
\hline $\mathrm{Cm}-247$ & 2.09E-10 & $0 \%$ & 2.08E-13 & $0 \%$ & $1.75 E-12$ & $0 \%$ & 120 & 2.09E-10 & $0 \%$ & $2.08 E-13$ & $0 \%$ & $1.75 E-12$ & $0 \%$ & 120 \\
\hline $\mathrm{Cm}-248$ & $1.05 E-10$ & $0 \%$ & $1.95 E-13$ & $0 \%$ & $1.64 E-12$ & $0 \%$ & 119 & 1.95E-10 & $0 \%$ & $1.95 E-13$ & $0 \%$ & $1.64 E-12$ & $0 \%$ & 118 \\
\hline Bk-249 & 2.88E-14 & $0 \%$ & $2.88 E-17$ & $0 \%$ & $1.33 E-16$ & $0 \%$ & 217 & $9.07 E-18$ & $0 \%$ & 9.07E-21 & $0 \%$ & $4.18 E-20$ & $0 \%$ & 217 \\
\hline Cf-249 & $1.60 E-12$ & $0 \%$ & $1.60 E-15$ & $0 \%$ & 1.34E-14 & $0 \%$ & 120 & $1.60 \mathrm{E}-12$ & $0 \%$ & $1.60 \mathrm{E}-15$ & $0 \%$ & $1.34 E-14$ & $0 \%$ & 120 \\
\hline Totel & $8.26 E+06$ & & $1.11 E+06$ & & $6.22 E+O 7$ & & & $B .01 E+08$ & & 6.07E+05 & & $4.44 E+07$ & & \\
\hline
\end{tabular}


RADKONUCLIDE DOSE CONTRIBUTION COMPARISON

Mark 318

\begin{tabular}{|c|c|c|c|c|c|c|c|c|c|c|c|c|c|c|}
\hline \multirow[b]{4}{*}{ Isotope } & \multirow{2}{*}{\multicolumn{4}{|c|}{ INHALATION }} & \multirow{2}{*}{\multicolumn{2}{|c|}{ INGESTION }} & \multirow{4}{*}{$\begin{array}{c}\text { Volatilo } \\
\text { Inh/lng } \\
\text { ratio }\end{array}$} & \multirow{2}{*}{\multicolumn{4}{|c|}{ INHALATION }} & \multirow{2}{*}{\multicolumn{2}{|c|}{ INGESTION }} & \multirow{4}{*}{$\begin{array}{c}\text { Volatile } \\
\text { Inh/ling } \\
\text { ratio }\end{array}$} \\
\hline & & & & & & & & & & & & & & \\
\hline & \multicolumn{2}{|c|}{ Volatile Conditions } & \multicolumn{2}{|c|}{ Evaporative Conditions } & \multicolumn{2}{|c|}{ Volatile Conditions } & & \multicolumn{2}{|c|}{ Volatile Conditions } & \multicolumn{2}{|c|}{ Evaporative Conditions } & \multicolumn{2}{|c|}{ Volatile Conditions } & \\
\hline & $\begin{array}{c}\text { Dose } \\
\text { (rem/g PU-239) }\end{array}$ & $\begin{array}{c}\text { Percent } \\
\text { Dose }\end{array}$ & $\begin{array}{c}\text { Dose } \\
\text { (rem/g Pu-239) }\end{array}$ & $\begin{array}{c}\text { Percent } \\
\text { Doses }\end{array}$ & $\begin{array}{c}\text { Dose } \\
(\mathrm{rem} / \mathrm{P} \mathrm{Pu}-239)\end{array}$ & $\begin{array}{l}\text { Percent } \\
\text { Dose }\end{array}$ & & $\begin{array}{c}\text { Dose } \\
\text { (rom/g Pu-239) }\end{array}$ & $\begin{array}{c}\text { Percent } \\
\text { Dose }\end{array}$ & $\begin{array}{c}\text { Dose } \\
\text { (rem/g Pu-239) }\end{array}$ & $\begin{array}{l}\text { Percent } \\
\text { Dose }\end{array}$ & $\begin{array}{c}\text { Dose } \\
\text { (rem/g Pu-239) }\end{array}$ & $\begin{array}{l}\text { Percent } \\
\text { Dose }\end{array}$ & \\
\hline H-3 & $0.00 E+\infty$ & $0 \%$ & $0.00 E+\infty$ & $0 \%$ & $0.00 \mathrm{E}+00$ & $0 \%$ & - & $0.00 E+\infty$ & $0 \%$ & $0.00 E+\infty$ & $0 \%$ & $0.00 E+\infty$ & $0 \%$ & - \\
\hline Cr-51 & 4.19E-16 & $0 \%$ & 4.19E-18 & $0 \%$ & $2.09 \mathrm{E}-16$ & $0 \%$ & 2 & $1.18 \mathrm{E}-55$ & $0 \%$ & $1.18 E-58$ & $0 \%$ & $5.89 \mathrm{E}-56$ & $0 \%$ & 2 \\
\hline co-60 & $4.64 E+05$ & $0 \%$ & $4.64 E+02$ & $0 \%$ & $8.03 E+04$ & $0 \%$ & 6 & $1.24 E+05$ & $0 \%$ & $1.24 E+02$ & $0 \%$ & $2.15 E+04$ & $0 \%$ & 6 \\
\hline So-78 & $1.83 E+01$ & $0 \%$ & $1.83 E-01$ & $0 \%$ & $1.64 E+01$ & $0 \%$ & 1 & $1.83 E+01$ & $0 \%$ & $1.83 \mathrm{E}-01$ & $0 \%$ & $1.64 E+01$ & $0 \%$ & 1 \\
\hline$R b-87$ & 2.01E-05 & $0 \%$ & 2.01E-05 & $0 \%$ & 2.92E-05 & $0 \%$ & 1 & 2.01E-05 & $0 \%$ & 2.01E-08 & $0 \%$ & 2.92E-05 & $0 \%$ & 1 \\
\hline Sr-89 & $5.96 \mathrm{E}-02$ & $0 \%$ & $5.96 E-05$ & $0 \%$ & 1.41E-02 & $0 \%$ & 4 & $4.28 E-23$ & $0 \%$ & 4.28E-26 & $0 \%$ & $1.01 \mathrm{E}-23$ & $0 \%$ & 4 \\
\hline St-90 & $5.29 E+08$ & 629 & $5.29 E+05$ & $46 \%$ & $5.29 E+07$ & $80 \%$ & 10 & $4.13 E+08$ & $68 \%$ & $4.13 E+05$ & $620 \%$ & $4.13 E+07$ & $87 \%$ & 10 \\
\hline$Y-90$ & 3.39E +06 & $0 \%$ & $3.39 E+03$ & $0 \%$ & $4.13 E+06$ & $6 \%$ & 1 & $2.65 E+06$ & $0 \%$ & $2.65 E+03$ & $0 \%$ & $3.23 E+06$ & $7 \%$ & 1 \\
\hline Y-91 & $1.24 E+\infty 0$ & $0 \%$ & $1.24 E-03$ & $0 \%$ & 2.5TE-01 & $0 \%$ & 5 & $2.15 E-18$ & $0 \%$ & $2.15 E-22$ & $0 \%$ & $4.33 E-20$ & $0 \%$ & 5 \\
\hline Zr-93 & $3.28 E+03$ & $0 \%$ & $3.28 E+\infty 0$ & $0 \%$ & $1.64 E+01$ & $0 \%$ & 200 & $3.28 E+03$ & $0 \%$ & $3.28 E+\infty 0$ & $0 \%$ & $1.64 E+01$ & $0 \%$ & 200 \\
\hline Zr-95 & $5.71 E+\infty 0$ & $0 \%$ & $5.71 E-03$ & $0 \%$ & $1.08 E+\infty 0$ & $0 \%$ & 5 & 9.34E-17 & $0 \%$ & $9.34 \mathrm{E}-20$ & $0 \%$ & 1.77E-17 & $0 \%$ & 5 \\
\hline $\mathrm{Nb}-95$ & $3.01 E+\infty$ & $0 \%$ & $3.01 E-03$ & $0 \%$ & $1.48 E+\infty 0$ & $0 \%$ & 2 & 4.92E-17 & $0 \%$ & $4.92 E-20$ & $0 \%$ & 2.41E-17 & $0 \%$ & 2 \\
\hline $\mathrm{Nb}-95 \mathrm{~m}$ & 8.37E-03 & $0 \%$ & 8.37E-06 & $0 \%$ & 7.61E-03 & $0 \%$ & 1 & $1.37 \mathrm{E}-19$ & $0 \%$ & 1.37E-22 & $0 \%$ & $1.25 E-19$ & $0 \%$ & 1 \\
\hline$T c-8 \theta$ & $0.00 E+00$ & $0 \%$ & $0.00 E+\infty$ & $0 \%$ & $0.00 E+\infty 0$ & $0 \%$ & - & $0.00 E+\infty 0$ & $0 \%$ & $0.00 E+\infty$ & $0 \%$ & $0.00 E+\infty$ & $0 \%$ & - \\
\hline Ru-103 & $4.38 E-06$ & $0 \%$ & $4.38 E-08$ & $0 \%$ & $1.52 E-06$ & $0 \%$ & 3 & 7.52E-34 & $0 \%$ & $7.52 E-36$ & $0 \%$ & $2.61 E-34$ & $0 \%$ & 3 \\
\hline Ru-106 & $3.14 E+07$ & $4 x$ & $3.14 E+05$ & $20 \%$ & $1.50 E+06$ & 28 & 21 & $3.30 E+04$ & $0 \%$ & $3.30 E+02$ & $0 \%$ & $1.58 E+\infty 3$ & $0 \%$ & 21 \\
\hline$P d-107$ & $6.12 E+\infty 0$ & $0 \%$ & $6.12 E-03$ & $\overline{0 \%}$ & 6.59E-02 & $0 \%$ & 83 & $6.12 E+\infty$ & $0 \%$ & $6.12 E-03$ & $0 \%$ & $6.60 \mathrm{E}-02$ & $0 \%$ & 93 \\
\hline Cd-115m & $3.92 E-06$ & $0 \%$ & 3.92E-09 & $0 \%$ & 8.04E-07 & $0 \%$ & 4 & $8.71 E-31$ & $0 \%$ & 8.71E-34 & $0 \%$ & $2.01 E-31$ & $0 \%$ & 4 \\
\hline $\ln -115$ & $7.81 E-07$ & $0 \%$ & $7.81 E-10$ & $0 \%$ & $3.21 E-08$ & $0 \%$ & 24 & 7.82E-07 & $0 \%$ & $7.82 E-10$ & $0 \%$ & $3.21 E-08$ & $0 \%$ & 24 \\
\hline $\mathrm{Sn}-121 \mathrm{~m}$ & $5.05 E+00$ & $0 \%$ & $5.05 E-03$ & $0 \%$ & 7.38E-01 & $0 \%$ & 7 & $4.40 E+\infty 0$ & $0 \%$ & 4.40E-03 & $0 \%$ & 6.42E-01 & $0 \%$ & 7 \\
\hline Sn-123 & $3.42 E+02$ & $0 \%$ & 3.42E-01 & $0 \%$ & $8.75 E+01$ & $0 \%$ & 4 & 1.02E-06 & $0 \%$ & 1.02E-09 & $0 \%$ & 2.63E-07 & $0 \%$ & 4 \\
\hline$S n-125$ & 2.31E-50 & $0 \%$ & 2.31E-50 & $0 \%$ & 1.73E-52 & $0 \%$ & 134 & $0.00 E+\infty 0$ & $0 \%$ & $0.00 E+\infty 0$ & $0 \%$ & $0.00 E+\infty$ & $0 \%$ & . \\
\hline$S_{n-126}$ & $3.35 E+02$ & $0 \%$ & 3.35E-01 & $0 \%$ & $6.62 E+01$ & $0 \%$ & 5 & $3.35 E+02$ & $0 \%$ & 3.35E-01 & $0 \%$ & $6.62 E+01$ & $0 \%$ & 5 \\
\hline$S b-124$ & 2.32E-05 & $0 \%$ & 2.32E-08 & $0 \%$ & $1.03 E-05$ & $0 \%$ & 2 & $1.27 E-23$ & $0 \%$ & 1.27E-26 & $0 \%$ & $5.63 \mathrm{E}-24$ & $0 \%$ & 2 \\
\hline Sb-125 & $2.78 E+05$ & $0 \%$ & $2.78 E+02$ & $0 \%$ & $7.41 E+04$ & $0 \%$ & 4 & $2.20 E+04$ & $0 \%$ & $2.20 E+01$ & $0 \%$ & $5.85 E+03$ & $0 \%$ & 4 \\
\hline$S b-126$ & $5.46 E-01$ & $0 \%$ & 5.46E-04 & $0 \%$ & $3.98 E-03$ & $0 \%$ & 137 & $5.46 \mathrm{E}-01$ & $0 \%$ & $5.46 E-04$ & $0 \%$ & $3.98 E-03$ & $0 \%$ & 137 \\
\hline To-127m & $7.18 E+01$ & $0 \%$ & 7.18E-01 & $0 \%$ & $2.99 E+01$ & $0 \%$ & 2 & 5.87E-09 & $0 \%$ & $5.87 E-11$ & $0 \%$ & 2.44E-09 & $0 \%$ & 2 \\
\hline To-128m & $3.73 E-09$ & $0 \%$ & $3.73 E-11$ & $0 \%$ & $1.84 E-09$ & $0 \%$ & 2 & 4.49E-42 & $0 \%$ & 4.49E-44 & $0 \%$ & $2.22 E-42$ & $0 \%$ & 2 \\
\hline $1-129$ & $0.00 E+\infty 0$ & $0 \%$ & $0.00 E+\infty 0$ & $0 \%$ & $0.00 \mathrm{E}+\infty 0$ & $0 \%$ & - & $0.00 E+\infty 0$ & $0 \%$ & $0.00 E+00$ & $0 \%$ & $0.00 E+00$ & $0 \%$ & - \\
\hline-131 & $0.00 E+\infty 0$ & $0 \%$ & $0.00 E+\infty 0$ & $0 \%$ & $0.00 E+\infty$ & $0 \%$ & - & $0.00 E+\infty 0$ & $0 \%$ & $0.00 E+\infty 0$ & $0 \%$ & $0.00 E+\infty$ & $0 \%$ & - \\
\hline Cs-134 & $2.73 E+04$ & $0 \%$ & $2.73 E+02$ & $0 \%$ & $4.30 E+04$ & $0 \%$ & 1 & $9.43 E+02$ & $0 \%$ & $9.43 E+\infty 0$ & $0 \%$ & $1.49 \mathrm{E}+03$ & $0 \%$ & 1 \\
\hline Ce-135 & 1.48E-01 & $0 \%$ & $1.48 E-03$ & $0 \%$ & 2.33E-01 & $0 \%$ & 1 & 1.48E-01 & $0 \%$ & $1.48 E-03$ & $0 \%$ & 2.34E-01 & $0 \%$ & 1 \\
\hline Cs-136 & 2.83E-37 & $0 \%$ & 2.83E-39 & $0 \%$ & $4.15 E-37$ & $0 \%$ & 1 & $0.00 E+\infty 0$ & $0 \%$ & $0.00 E+\infty$ & $0 \%$ & $0.00 E+\infty 0$ & $0 \%$ & - \\
\hline C2-137 & $8.42 E+05$ & $0 \%$ & $8.42 E+03$ & $1 x$ & $1.32 E+06$ & 2\% & 1 & $6.69 E+05$ & $0 \%$ & $6.68 E+03$ & $1 \%$ & $1.05 E+06$ & $2 \%$ & 1 \\
\hline$B=-140$ & $7.78 E-35$ & $0 \%$ & 7.78E-38 & $0 \%$ & 1.81E-34 & $0 \%$ & 0 & $0.00 E+\infty 0$ & $0 \%$ & $0.00 E+00$ & $0 \%$ & $0.00 E+\infty$ & $0 \%$ & - \\
\hline Lo- 140 & $1.08 E-34$ & $0 \%$ & $1.095-37$ & $0 \%$ & $1.91 E-34$ & $0 \%$ & 1 & $0.00 E+\infty 0$ & $0 \%$ & $0.00 E+\infty 0$ & $0 \%$ & $0.00 E+\infty 0$ & $0 \%$ & . \\
\hline Co-141 & 1.32E-08 & $0 \%$ & 1.32E-11 & $0 \%$ & 4.03E-09 & $0 \%$ & 3 & $2.09 E-42$ & $0 \%$ & $2.09 E-45$ & $0 \%$ & $6.39 E-43$ & $0 \%$ & 3 \\
\hline$C_{0-144}$ & $6.86 E+07$ & Bx & $6.86 \mathrm{E}+04$ & 6x & $3.96 E+06$ & 6\% & 18 & $8.47 E+03$ & $0 \%$ & $9.47 E+\infty 0$ & $0 \%$ & $5.40 \mathrm{E}+02$ & $0 \%$ & 18 \\
\hline Pr-143 & $4.63 E-32$ & $0 \%$ & 4.83E-35 & $0 \%$ & $2.98 \mathrm{E}-32$ & $0 \%$ & 2 & $0.00 E+\infty 0$ & $0 \%$ & $0.00 E+\infty 0$ & $0 \%$ & $0.00 \mathrm{E}+\infty 0$ & $0 \%$ & \\
\hline$N d-144$ & $0.00 E+\infty 0$ & $0 \%$ & $0.00 E+\infty 0$ & $0 \%$ & $0.00 E+\infty 0$ & $0 \%$ & - & $0.00 E+\infty 0$ & $0 \%$ & $0.00 E+\infty 0$ & $0 \%$ & $0.00 \mathrm{E}+\infty$ & $0 \%$ & - \\
\hline$N d-147$ & $4.74 E-42$ & $0 \%$ & $4.74 E-45$ & $0 \%$ & $2.98 E-42$ & $0 \%$ & 2 & $0.00 E+\infty 0$ & $0 \%$ & $0.00 E+\infty$ & $0 \%$ & $0.00 E+\infty 0$ & $0 \%$ & - \\
\hline$P m-147$ & $2.04 E+07$ & 24 & $2.04 E+04$ & 20 & $5.72 E+05$ & 18 & 36 & $1.46 E+06$ & $0 \%$ & $1.46 E+03$ & $0 \%$ & $4.07 E+04$ & $0 \%$ & 36 \\
\hline
\end{tabular}


RADKONUCLIDE DOSE CONTRIBUTION COMPARISON

Mark 318

\begin{tabular}{|c|c|c|c|c|c|c|c|c|c|c|c|c|c|c|}
\hline \multirow[b]{4}{*}{ Isotope } & \multirow{3}{*}{\multicolumn{4}{|c|}{$\begin{array}{c}\text { INHALATION } \\
\text { INATH }\end{array}$}} & \multirow{3}{*}{\multicolumn{2}{|c|}{$\begin{array}{l}\text { INGESTION } \\
\text { Volatile Conditions }\end{array}$}} & \multirow{4}{*}{$\begin{array}{c}\text { Volatile } \\
\text { Inh/lng } \\
\text { ratio }\end{array}$} & \multirow{3}{*}{\multicolumn{4}{|c|}{$\begin{array}{c}\text { INHALATION } \\
\text { Volations }\end{array}$}} & \multirow{2}{*}{\multicolumn{2}{|c|}{ INGESTION }} & \multirow{4}{*}{$\begin{array}{c}\text { Volatite } \\
\text { Inh//ng } \\
\text { ratio }\end{array}$} \\
\hline & & & & & & & & & & & & & & \\
\hline & & & \multicolumn{2}{|c|}{ Evaporative Conditions } & & & & & & & & Volatile Cor & ditions & \\
\hline & $\begin{array}{c}\text { Dose } \\
\text { (rem/g Pu-239) }\end{array}$ & $\begin{array}{c}\text { Percent } \\
\text { Dose }\end{array}$ & $\begin{array}{c}\text { Dose } \\
(r \in m / g \text { Pu-239) }\end{array}$ & $\begin{array}{c}\text { Percent } \\
\text { Dose }\end{array}$ & $\begin{array}{c}\text { Dose } \\
(r \in m / g \text { Pu-239) }\end{array}$ & $\begin{array}{c}\text { Percent } \\
\text { Dose }\end{array}$ & & $\begin{array}{c}\text { Dose } \\
\text { (rem/g Pu-239) }\end{array}$ & $\begin{array}{c}\text { Percent } \\
\text { Dose }\end{array}$ & $\begin{array}{c}\text { Dose } \\
\text { (rem/g Pu-239) }\end{array}$ & $\begin{array}{c}\text { Percent } \\
\text { Dose }\end{array}$ & $\begin{array}{c}\text { Dose } \\
\text { (rom/g Pu-239) }\end{array}$ & $\begin{array}{c}\text { Percent } \\
\text { Dose }\end{array}$ & \\
\hline$P \pi-148$ & $1.07 E-08$ & $0 \%$ & 1.07E-11 & $0 \%$ & $1.02 \mathrm{E}-08$ & $0 \%$ & 1 & $2.56 \mathrm{E}-35$ & $0 \%$ & $2.56 \mathrm{E}-38$ & $0 \%$ & $2.43 \mathrm{E}-35$ & $0 \%$ & 1 \\
\hline$P m-148 m$ & $1.56 \mathrm{E}-07$ & $0 \%$ & $1.56 E-10$ & $0 \%$ & $1.09 E-07$ & $0 \%$ & 1 & $3.72 E-34$ & $0 \%$ & 3.72E-37 & $0 \%$ & 2.61E-34 & $0 \%$ & 1 \\
\hline Sm-147 & $2.95 E+\infty 0$ & $0 \%$ & $2.95 E-03$ & $0 \%$ & $7.49 \mathrm{E}-03$ & $0 \%$ & 394 & $3.95 E+\infty$ & $0 \%$ & 3.95E-03 & $0 \%$ & $1.00 \mathrm{E}-02$ & $0 \%$ & 394 \\
\hline$S m-151$ & $2.40 E+05$ & $0 \%$ & $2.40 E+02$ & $0 \%$ & $2.82 E+03$ & $0 \%$ & 85 & $2.23 E+05$ & $0 \%$ & $2.23 E+02$ & $0 \%$ & $2.61 E+03$ & $0 \%$ & 85 \\
\hline Eu-152 & $2.57 E+04$ & $0 \%$ & $2.57 \mathrm{E}+01$ & $0 \%$ & $7.02 E+02$ & $0 \%$ & 37 & $1.51 E+04$ & $0 \%$ & $1.51 E+01$ & $0 \%$ & $4.12 E+02$ & $0 \%$ & 37 \\
\hline Eu-154 & $8.39 E+05$ & $0 \%$ & $8.39 E+02$ & $0 \%$ & $2.94 E+04$ & $0 \%$ & 29 & $3.75 E+05$ & $0 \%$ & $3.75 E+02$ & $0 \%$ & $1.31 E+04$ & $0 \%$ & 29 \\
\hline Eu-155 & $6.04 E+05$ & $0 \%$ & $6.04 E+02$ & $0 \%$ & $2.02 E+04$ & $0 \%$ & 30 & $1.42 E+05$ & $0 \%$ & $1.42 E+02$ & $0 \%$ & $4.76 E+03$ & $0 \%$ & 30 \\
\hline Eu-156 & $3.60 E-29$ & $0 \%$ & $3.60 E-32$ & $0 \%$ & $2.85 E-29$ & $0 \%$ & 1 & $0.00 E+\infty 0$ & $0 \%$ & $0.00 E+\infty 0$ & $0 \%$ & $0.00 E+00$ & $0 \%$ & $\cdot$ \\
\hline Gd-152 & $9.73 E-08$ & $0 \%$ & 9.73E-11 & $0 \%$ & $6.08 E-11$ & $0 \%$ & 1599 & $9.73 E-08$ & $0 \%$ & $9.73 \mathrm{E}-11$ & $0 \%$ & $6.08 E-11$ & $0 \%$ & 1599 \\
\hline$T \mathrm{~b}-160$ & $5.38 E-04$ & $0 \%$ & $5.38 E-07$ & $0 \%$ & 1.57E-04 & $0 \%$ & 3 & $3.34 \mathrm{E}-19$ & $0 \%$ & 3.34E-22 & $0 \%$ & $9.72 E-20$ & $0 \%$ & 3 \\
\hline Pa-224 & $4.22 E+01$ & $0 \%$ & 4.22E-02 & $0 \%$ & $4.80 E+\infty 0$ & $0 \%$ & 8 & $2.75 E+01$ & $0 \%$ & 2.75E-02 & $0 \%$ & $3.13 E+\infty 0$ & $0 \%$ & 9 \\
\hline Th-228 & $4.45 E+03$ & $0 \%$ & $4.45 E+\infty$ & $0 \%$ & $5.45 E+00$ & $0 \%$ & 817 & $2.91 E+03$ & $0 \%$ & $2.91 E+\infty$ & $0 \%$ & $3.56 E+\infty 0$ & $0 \%$ & 817 \\
\hline U.232 & $4.82 E+03$ & $0 \%$ & $4.82 E+00$ & $0 \%$ & $9.32 E+\infty$ & $0 \%$ & 517 & $6.46 E+03$ & $0 \%$ & $6.46 \mathrm{E}+\infty 0$ & $0 \%$ & $1.25 E+01$ & $0 \%$ & 517 \\
\hline U-234 & $1.40 E-11$ & $0 \%$ & $1.40 \mathrm{E}-14$ & $0 \%$ & $2.81 E-14$ & $0 \%$ & 499 & $1.40 E-11$ & $0 \%$ & 1.40E-14 & $0 \%$ & $2.81 E-14$ & $0 \%$ & 499 \\
\hline U-235 & $3.68 E+01$ & $0 \%$ & $3.68 E-02$ & $0 \%$ & 7.69E-02 & $0 \%$ & 479 & $3.68 E+01$ & $0 \%$ & 3.68E-02 & $0 \%$ & 7.69E-02 & $0 \%$ & 479 \\
\hline U-236 & $3.82 E+01$ & $0 \%$ & $3.82 E-02$ & $0 \%$ & $7.98 E-02$ & $0 \%$ & 479 & $3.82 E+01$ & $0 \%$ & $3.82 E-02$ & $0 \%$ & $7.98 \mathrm{E}-02$ & $0 \%$ & 478 \\
\hline U-238 & $3.44 E+03$ & $0 \%$ & $3.44 E+00$ & $0 \%$ & $6.60 E+\infty$ & $0 \%$ & 521 & $3.44 E+03$ & $0 \%$ & $3.44 E+\infty 0$ & $0 \%$ & $6.60 E+\infty 0$ & $0 \%$ & 521 \\
\hline Np-237 & $4.61 E+03$ & $0 \%$ & $4.61 E+\infty 0$ & $0 \%$ & $3.67 E+01$ & $0 \%$ & 125 & $4.61 E+03$ & $0 \%$ & $4.61 E+\infty$ & $0 \%$ & 3.67E+01 & $0 \%$ & 125 \\
\hline Pu-236 & $1.43 E+04$ & $0 \%$ & $1.43 E+01$ & $0 \%$ & $1.16 E+02$ & $0 \%$ & 123 & $1.26 E+03$ & $0 \%$ & $1.26 E+00$ & $0 \%$ & $1.02 E+01$ & $0 \%$ & 123 \\
\hline Pu-238 & $9.42 E+05$ & $0 \%$ & $9.42 E+02$ & $0 \%$ & $7.78 E+03$ & $0 \%$ & 121 & $8.81 E+05$ & $0 \%$ & $8.81 E+02$ & $0 \%$ & $7.28 E+03$ & $0 \%$ & 121 \\
\hline Pu-239" & $3.16 E+07$ & 48 & $3.16 E+04$ & $3 \%$ & $2.67 E+05$ & $0 \%$ & 118 & $3.16 E+07$ & $5 \%$ & $3.16 E+04$ & $5 \%$ & $2.67 E+05$ & $1 \%$ & 118 \\
\hline Pu-240 & $7.08 E+06$ & $1 \%$ & $7.08 E+03$ & 18 & $5.95 E+0.4$ & $0 \%$ & 119 & $7.08 E+06$ & $1 \%$ & $7.08 E+03$ & 18 & $5.95 E+04$ & $0 \%$ & 119 \\
\hline Pu-241 & $5.9 \pi E+06$ & $1 \%$ & $5.97 E+03$ & 18 & $5.13 E+04$ & $0 \%$ & 116 & $3.72 E+06$ & $1 \%$ & $3.72 E+06$ & 14. & $3.20 E+04$ & $0 \%$ & 116 \\
\hline Pu-242 & $5.05 E+02$ & $0 \%$ & 5.05E- -01 & $0 \%$ & $4.30 E+\infty 0$ & $0 \%$ & 117 & $5.05 E+02$ & $0 \%$ & $5.05 E-01$ & $0 \%$ & $4.30 E+00$ & $0 \%$ & 117 \\
\hline Am-241 & $1.46 E+08$ & $17 \%$ & $1.46 E+05$ & $13 x$ & $1.26 E+06$ & 2\% & 116 & $1.48 E+08$ & 24\% & $1.48 E+05$ & $24 \%$ & $1.27 E+06$ & $3 \%$ & 116 \\
\hline Am-242m & $1.80 E+05$ & $0 \%$ & $1.80 E+02$ & $0 \%$ & $1.48 E+03$ & $0 \%$ & 122 & $1.72 E+05$ & $0 \%$ & $1.72 E+02$ & $0 \%$ & $1.41 E+03$ & $0 \%$ & 122 \\
\hline Am-243 & $4.2 \pi+02$ & $0 \%$ & 4.27E-01 & $0 \%$ & $3.68 E+\infty 0$ & $0 \%$ & 116 & $4.26 E+02$ & $0 \%$ & 4.26E-01 & $0 \%$ & $3.68 E+\infty$ & $0 \%$ & 116 \\
\hline $\mathrm{Cm}-242$ & $1.08 E+04$ & $0 \%$ & $1.09 E+01$ & $0 \%$ & $7.06 E+01$ & $0 \%$ & 155 & $4.71 E+03$ & $0 \%$ & $4.71 E+\infty 0$ & $0 \%$ & $3.05 E+01$ & $0 \%$ & 154 \\
\hline $\mathrm{Cm}-243$ & $7.13 E+03$ & $0 \%$ & 7.13E+00 & $0 \%$ & $5.91 E+01$ & $0 \%$ & 121 & $5.59 E+03$ & $0 \%$ & $5.59 E+\infty 0$ & $0 \%$ & $4.64 E+01$ & $0 \%$ & 121 \\
\hline $\mathrm{Cm}-244$ & 1.19E+05 & $0 \%$ & $1.19 E+02$ & $0 \%$ & $1.02 E+03$ & $0 \%$ & 117 & $8.14 E+02$ & $0 \%$ & 8.14E-01 & $0 \%$ & $6.94 E+\infty$ & $0 \%$ & 117 \\
\hline $\mathrm{Cm}-245$ & 2.27E-02 & $0 \%$ & $2.27 E-05$ & $0 \%$ & $1.89 E-04$ & $0 \%$ & 120 & 2.27E-02 & $0 \%$ & $2.27 E-05$ & $0 \%$ & $1.89 \mathrm{E}-04$ & $0 \%$ & 120 \\
\hline $\mathrm{Cm}-246$ & $4.18 E-04$ & $0 \%$ & $4.18 E-07$ & $0 \%$ & $3.48 E-06$ & $0 \%$ & 120 & $4.17 E-04$ & $0 \%$ & 4.17E-07 & $0 \%$ & $3.49 \mathrm{E}-0.6$ & $0 \%$ & 120 \\
\hline $\mathrm{Cm}-247$ & 9.2BE-11 & $0 \%$ & $9.28 E-14$ & $0 \%$ & $7.75 E-13$ & $0 \%$ & 120 & $9.28 E-11$ & $0 \%$ & $9.28 \mathrm{E}-14$ & $0 \%$ & 7.75E-13 & $0 \%$ & 120 \\
\hline $\mathrm{Cm}-248$ & 7.26E-11 & $0 \%$ & $7.26 E-14$ & $0 \%$ & $6.11 E-13$ & $0 \%$ & 119 & $7.26 E-11$ & $0 \%$ & $7.26 E-14$ & $0 \%$ & $6.11 E-13$ & $0 \%$ & 118 \\
\hline Bk-249 & 1.10E-14 & $0 \%$ & 1.10E-17 & $0 \%$ & $5.07 E-17$ & $0 \%$ & 217 & $3.47 E-18$ & $0 \%$ & 3.47E-21 & $0 \%$ & $1.60 \mathrm{E}-20$ & $0 \%$ & 217 \\
\hline$C f-249$ & 6.13E-13 & $0 \%$ & $6.13 E-16$ & $0 \%$ & 5.13E-15 & $0 \%$ & 120 & $6.12 E-13$ & $0 \%$ & $6.12 E-16$ & $0 \%$ & 5.12E-15 & $0 \%$ & 120 \\
\hline Total & $8.49 E+06$ & & $1.14 E+06$ & & $6.63 E+07$ & & & $6.10 E+08$ & & $4.33 E+06$ & & A.73E+07 & & \\
\hline
\end{tabular}


WSRC-TR-94-0262

Rev. 0

June 28, 1994

DISTRIBUTION:

T. M. Monahon, 703-H

V. G. Dickert, 703-H

J. E. Marra, 703-H

M. C. Chandler, 703-H

C. E. Bess, 703-H

C. I. Aponte, 703-H

P. D. d'Entremont, 703-H

J. D. Carlson, 703-H

S. Mehta, 703-H

HLW Files, 703-H, File Code 176.1

Document Control, 703-H 